\title{
Fast, Sound, and Effectively Complete Dynamic Race Prediction
}

\author{
ANDREAS PAVLOGIANNIS, Aarhus University, Denmark
}

Writing concurrent programs is highly error-prone due to the nondeterminism in interprocess communication. The most reliable indicators of errors in concurrency are data races, which are accesses to a shared resource that can be executed concurrently. We study the problem of predicting data races in lock-based concurrent programs. The input consists of a concurrent trace $t$, and the task is to determine all pairs of events of $t$ that constitute a data race. The problem lies at the heart of concurrent verification and has been extensively studied for over three decades. However, existing polynomial-time sound techniques are highly incomplete and can miss simple races.

In this work we develop M2: a new polynomial-time algorithm for this problem, which has no false positives. In addition, our algorithm is complete for input traces that consist of two processes, i.e., it provably detects all races in the trace. We also develop sufficient criteria for detecting completeness dynamically in cases of more than two processes. We make an experimental evaluation of our algorithm on a challenging set of benchmarks taken from recent literature on the topic. Our algorithm soundly reports hundreds of real races, many of which are missed by existing methods. In addition, using our dynamic completeness criteria, M2 concludes that it has detected all races in the benchmark set, hence the reports are both sound and complete. Finally, its running times are comparable, and often smaller than the theoretically fastest, yet highly incomplete, existing methods. To our knowledge, M2 is the first sound algorithm that achieves such a level of performance on both running time and completeness of the reported races.

CCS Concepts: • Software and its engineering $\rightarrow$ Software verification and validation; ・ Theory of computation $\rightarrow$ Theory and algorithms for application domains; Program analysis.

Additional Key Words and Phrases: concurrency, race detection, predictive analyses

\section{ACM Reference Format:}

Andreas Pavlogiannis. 2020. Fast, Sound, and Effectively Complete Dynamic Race Prediction. Proc. ACM Program. Lang. 4, POPL, Article 17 (January 2020), 29 pages. https://doi.org/10.1145/3371085

\section{INTRODUCTION}

Verification of concurrent programs. Writing concurrent software is notoriously hard due to the inherent nondeterminism in the way that accesses to shared resources are scheduled. Accounting for all possible nondeterministic choices is hard, even to experienced developers. This makes the development of concurrent software prone to concurrency bugs [Lu et al. 2008; Shi et al. 2010], i.e., bugs that are present only in a few among the (possibly exponentially) many executions of the program. Since developers have no control over the scheduler, concurrency bugs are also

Author's address: Andreas Pavlogiannis, Aarhus University, Aabogade 34, Aarhus, 8200, Denmark, pavlogiannis@cs.au.dk.

This work is licensed under a Creative Commons Attribution 4.0 International License.

(C) 2020 Copyright held by the owner/author(s).

2475-1421/2020/1-ART17

https://doi.org/10.1145/3371085

Proc. ACM Program. Lang., Vol. 4, No. POPL, Article 17. Publication date: January 2020. 
hard to reproduce by testing (often categorized as Heisenbugs [Gray 1985; Musuvathi et al. 2008]). Consequently, testing alone is considered an ineffective approach for detecting bugs in concurrent programs. To circumvent this difficulty, testing techniques are often combined with model checking. First, a testing phase produces a set of concrete program executions. Then, a verification phase makes a formal treatment of these executions and identifies whether there exist other "neighboring" executions that are not present in the test set but (i) constitute valid executions of the program and (ii) manifest a bug. Hence, even though the scheduler might "hide" a bug in the test set, this bug can be effectively caught by formal techniques applied on the test set.

Data races. Two events $\left(e_{1}, e_{2}\right)$ of a concurrent program are called conflicting if they access the same shared resource (e.g., the same global variable $x$ ) and at least one of them modifies the resource (e.g., writes to $x$ ). A data race is typically defined as a conflicting pair $\left(e_{1}, e_{2}\right)$ that can be executed concurrently [Bond et al. 2010; Flanagan and Freund 2009; Helmbold et al. 1991; O'Callahan and Choi 2003]. Data races are the prime suspects of erroneous behavior, and there have been significant efforts spanning across several decades towards detecting data races efficiently, starting with seminal papers found in [Dinning and Schonberg 1991; Helmbold et al. 1991; Schonberg 1989].

Dynamic race detection. Dynamic algorithms for race detection operate on a single execution (i.e., a trace) of the concurrent program, and their task is to identify pairs of events of the trace that constitute a race, even though the race might not be manifested in the input trace. Dynamic race detection is a popular technique that combines testing with formal reasoning. Existing dynamic algorithms typically fall into one of the following three categories.

Lockset-based techniques [Dinning and Schonberg 1991; Elmas et al. 2007; Savage et al. 1997] report races by comparing the sets of locks which guard conflicting data accesses. This approach typically reports spurious races, as data accesses protected by different locks can nevertheless be separated by other control-flow and data dependencies, and thus not constitute a race.

Exhaustive predictive-runtime techniques [Chen and Roşu 2007; Huang et al. 2014; Said et al. 2011; Sen et al. 2005] report races by exploring all possible valid reorderings of the input trace. These techniques typically rely on SAT/SMT solvers and are sound and complete in theory; however, as there are exponentially many valid reorderings, they have exponential complexity. In practice, completeness is traded for runtime, by using windowing techniques which slice the input trace into small fragments and analyze each fragment separately.

Partial-order-based techniques are probably the most well-known and widely-used. The underlying principle is to construct a partial order $P$ on the events of the input trace. Afterwards, a race is reported between a pair of events if the two events are unordered by $P$. These techniques are usually efficient, as constructing the partial order typically requires polynomial time. However, in order for $P$ to admit a linearization to a valid witness trace that exposes the race, $P$ enforces many arbitrary orderings between events. These arbitrary orderings often result in an ordering between the events of an actual race, and thus $P$ misses the race.

Most of the above techniques are based on Lamport's happens-before (HB) partial order [Lamport 1978] which is implemented in various tools [Bond et al. 2010; Christiaens and Bosschere 2001; Flanagan and Freund 2009; Pozniansky and Schuster 2003; Schonberg 1989; Yu et al. 2005]. As HB is highly incomplete, there have been several efforts for constructing weaker partial orders that are efficiently computable, such as the causally-precedes partial order CP [Smaragdakis et al. 2012]. Partial-order techniques recently led to important advances in predictive race detection, based on the weakly-causally-precedes WCP [Kini et al. 2017], schedulably-happens-before SHB [Mathur 
et al. 2018] and doesn't-commute DC [Roemer et al. 2018] partial orders. We next discuss these approaches in more detail and outline the motivation behind our work.

\subsection{Motivating Examples}

We illustrate the motivation behind our work with a few simple examples (Figure 1) which highlight some completeness issues that the existing approaches based on HB, WCP and DC partial orders suffer from. We focus on single races here, in which case SHB is subsumed by HB. We remark that we focus on polynomial-time, sound methods here, and hence we do not consider unsound techniques (e.g., lockset-based [Savage et al. 1997]) or techniques that rely on SAT/SMT solvers and are thus not polynomial time (e.g., [Huang et al. 2014]). In each example, we use the notation $\tau_{i}$ to refer to the local trace of the $i$-th process, and $e_{j}$ to refer to the $j$-th event in the concurrent trace. We note that the underlying memory model is sequentially consistent, i.e., in every trace, a read event observes the value of the last write event that writes to the location read by the read event.

To develop some context, we briefly outline how each of these techniques works by ordering events of the input trace. We refer to Appendix B for the formal definitions. In all cases, events that belong to the same process are always totally ordered according to their order in the input trace.

(1) The HB and WCP techniques operate in a similar manner. They perform a single pass of $t$ and construct a partial order $\leq_{\mathrm{HB}}\left(\right.$ resp., $\left.\leq_{\mathrm{WCP}}\right)$. A race $\left(e, e^{\prime}\right)$ is reported if $e, e^{\prime}$ are conflicting and $e \not_{\mathrm{HB}} e^{\prime}$ (resp., $e \Varangle_{\mathrm{WCP}} e^{\prime}$ ), i.e., the two events are unordered by the respective partial order.

(2) $\mathrm{DC}$ operates in three phases, which all have to succeed for $\left(e, e^{\prime}\right)$ to be reported as a race.

(a) In Phase 1, a DC partial order is constructed, similarly to HB and WCP. If $e \leq_{\mathrm{DC}} e^{\prime}$ then $\left(e, e^{\prime}\right)$ is reported as a non-race.

(b) In Phase 2, a constraint graph $G$ is constructed which contains the DC orderings. Then, more ordering constraints are inserted in $G$. If $G$ becomes cyclic during this process, $\left(e, e^{\prime}\right)$ is reported as a non-race. If $t^{*}$ fails to respect lock semantics, $\left(e, e^{\prime}\right)$ is reported as a non-race.

\begin{tabular}{l|l|l|} 
& $\tau_{1}$ & $\tau_{2}$ \\
\hline 1 & $\operatorname{acq}(\ell)$ & \\
2 & $\mathbf{w}(\mathbf{x})$ & \\
3 & $\operatorname{rel}(\ell)$ & \\
4 & & $\operatorname{acq}(\ell)$ \\
5 & & $w(x)$ \\
6 & & $\operatorname{rel}(\ell)$ \\
7 & & $\mathbf{r}(\mathbf{x})$ \\
\hline
\end{tabular}

(a) Is $\left(e_{2}, e_{7}\right)$ a race?

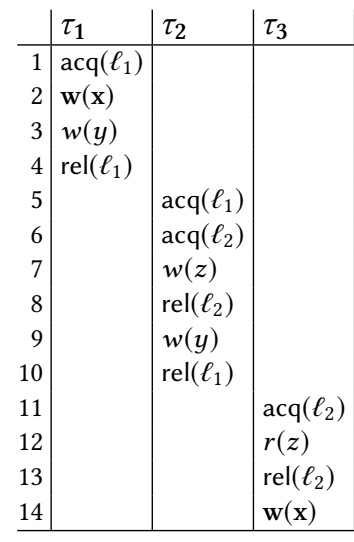

(b) Is $\left(e_{2}, e_{14}\right)$ a race?

Fig. 1. Examples in which $\mathrm{HB}, \mathrm{WCP}$ and $\mathrm{DC}$ are incomplete. (a) A race $\left(e_{2}, e_{7}\right)$ missed by HB, WCP and DC. (b) A race $\left(e_{2}, e_{14}\right)$ missed by HB, WCP and DC (in Phase 2 ).

Incompleteness. Each of $\mathrm{HB}, \mathrm{WCP}$ and DC methods are incomplete i.e., the input trace $t$ can have arbitrarily many predictable races, however each of these methods falsely reports that there is no race in $t$. We present a couple of examples where HB, WCP and DC fail to detect simple races. 
Figure 1a. There is a predictable race $\left(e_{2}, e_{7}\right)$. HB defines $e_{3} \leq_{\mathrm{HB}} e_{4}$, and thus $e_{2} \leq_{\mathrm{HB}} e_{7}$, hence missing the race. Similarly, WCP (resp., DC) defines $e_{3} \leq \mathrm{WCP} e_{5}$ (resp., $e_{3} \leq_{\mathrm{DC}} e_{5}$ ) and thus $e_{2} \leq \mathrm{WCP} e_{7}$ (resp., $e_{2} \leq_{\mathrm{DC}} e_{7}$ ), hence missing the race. Intuitively, WCP and DC fail to swap the two critical sections because they contain the conflicting events $w(x)$. Note that here DC fails in Phase 1 . However, $\left(e_{2}, e_{7}\right)$ is a true race that is detected by the techniques developed in this work, exposed by the witness trace $t^{*}=e_{4}, e_{5}, e_{6}, e_{1}, e_{2}, e_{7}$.

Figure $1 b$. There is a predictable race $\left(e_{2}, e_{14}\right)$. HB defines $e_{4} \leq_{\mathrm{HB}} e_{5}$ and $e_{8} \leq_{\mathrm{HB}} e_{11}$, and thus $e_{2} \leq_{\mathrm{HB}} e_{14}$, hence missing the race. Similarly, WCP defines $e_{4} \leq_{\mathrm{WCP}} e_{5}$ and $e_{8} \leq_{\mathrm{WCP}} e_{12}$ and thus $e_{2} \leq$ WCP $e_{14}$, hence missing the race. Intuitively, WCP fails to swap the critical sections of $\tau_{1}$ and $\tau_{2}$ on $\ell_{1}$ because WCP is closed under composition with $\mathrm{HB}$, and in turn $\mathrm{HB}$ totally orders critical sections as in the input trace. On the other hand, DC does not compose with $\mathrm{HB}$, and the only enforced orderings are $e_{4} \leq_{\mathrm{DC}} e_{9}$ and $e_{8} \leq_{\mathrm{DC}} e_{12}$. Hence DC proceeds with Phase 2, where it constructs a constraint graph $G$. Since $e_{4} \leq_{\mathrm{DC}} e_{9}$ and $e_{9}$ belongs in a critical section on lock $\ell_{1}$ which is released by $e_{4}$, in order to not violate lock semantics, $G$ forces the ordering $e_{4} \rightsquigarrow e_{5}$. In addition, $G$ forces the ordering $e_{5} \rightsquigarrow e_{2}$, since $e_{2}$ is the racy event and must appear last in the witness trace. Note that this creates a cycle and hence DC fails in Phase 2 . However, $\left(e_{2}, e_{14}\right)$ is a true race that is detected by the techniques developed in this work, exposed by the witness trace $t^{*}=e_{5}, e_{6}, e_{7}, e_{8}, e_{9}, e_{10}, e_{11}, e_{12}, e_{13}, e_{1}, e_{2}, e_{14}$.

Algorithmic challenge. We have seen that state-of-the-art approaches fail to catch simple races. Intuitively, the algorithmic challenge that underlies race detection is that of constructing a partial order $P$ with the following properties.

(1) $P$ is as weak as possible, so that a race $\left(e_{i}, e_{j}\right)$ remains unordered in $P$.

(2) $P$ is efficiently linearizable to a valid trace that exposes the race.

These two features are opposing each other, as the weaker the partial order, the more linearizations it admits, and finding a valid one becomes harder.

Our approach. In this work we develop a new predictive technique for race detection. At its core, our algorithm constructs partial orders that are much weaker than existing approaches (hence detecting more races), while these partial orders are efficiently (polynomial-time) linearizable to valid traces (hence the reported races are exposed efficiently). To give a complete illustration of our insights, we use the more involved example in Figure 2.

The task is to decide whether $\left(e_{10}, e_{19}\right)$ is a predictable race of the input trace $t$ (Figure 2a). To keep the presentation simple, we ignore the other data races that occur, which can be trivially avoided by inserting additional lock events. Note that HB, WCP and DC report no race in $t$, as they all order $e_{11} \leq e_{14}$. In order to detect this race, we need to make some non-trivial reasoning about reordering certain events in $t$. Our reasoning can be summarized in the following steps.

(1) If $\left(e_{10}, e_{19}\right)$ is a race of $t$, a witness trace $t^{*}$ can be constructed in which both $e_{10}$ and $e_{19}$ are the last events. Observe that $t^{*}$ will not contain the rel $(\ell)$ event $e_{11}$.

(2) Since we ignore event $e_{11}$, that critical section of $\tau_{1}$ remains open in $t^{*}$. Hence the rel $(\ell)$ event $e_{15}$ must be ordered before the acq $(\ell)$ event $e_{8}$. In addition, the $w\left(x_{2}\right)$ event $e_{2}$ is observed by the $r\left(x_{2}\right)$ event $e_{17}$, hence $e_{2}$ must be ordered before $e_{17}$. These constraints, together with the program order which requires events of each process to occur in the same order as in the input trace, are captured by the partial order shown in solid edges in Figure 2b. Note that several conflicting accesses to $x_{1}, x_{3}$ and $x_{4}$ are still unordered. How can we obtain a valid linearization? First, we can infer a few more orderings. 


\begin{tabular}{r|l|l|} 
& $\tau_{1}$ & $\tau_{2}$ \\
\hline 1 & $w\left(x_{1}\right)$ & \\
2 & $w\left(x_{2}\right)$ & \\
3 & $w\left(x_{3}\right)$ & \\
4 & $\operatorname{acq}(\ell)$ & \\
5 & $w\left(x_{4}\right)$ & \\
6 & $r\left(x_{1}\right)$ & \\
7 & $\operatorname{rel}(\ell)$ & \\
8 & $\operatorname{acq}(\ell)$ & \\
9 & $r\left(x_{4}\right)$ & \\
10 & $\mathbf{w}(\mathbf{x})$ & \\
11 & $\operatorname{rel}(\ell)$ & \\
12 & & $w\left(x_{3}\right)$ \\
13 & & $\operatorname{acq}(\ell)$ \\
14 & & $w\left(x_{4}\right)$ \\
15 & & $\operatorname{rel}(\ell)$ \\
16 & & $w\left(x_{1}\right)$ \\
17 & & $r\left(x_{2}\right)$ \\
18 & & $r\left(x_{3}\right)$ \\
19 & & $\mathbf{r}(\mathbf{x})$ \\
\hline
\end{tabular}

(a) Is $\left(e_{10}, e_{19}\right)$ a race?

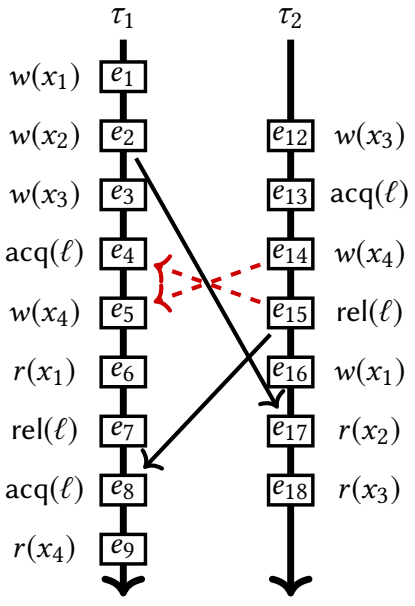

(b) Ordering constraints before (solid edges) and after the closure (solid and dashed edges).

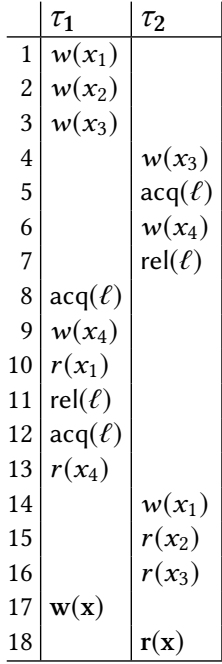

(c) The witness trace.

Fig. 2. Example of a race that requires non-trivial reasoning about reorderings of the input trace.

(3) The $r\left(x_{4}\right)$ event $e_{9}$ must observe the same write event as in $t$. Due to the previous step, the $w\left(x_{4}\right)$ event $e_{14}$ now is ordered before $e_{9}$. To avoid $e_{9}$ observing $e_{14}$, we perform an observation-closure step, by ordering $e_{14}$ before the observation $e_{5}$ of $e_{9}$ (see dashed edge in Figure $2 \mathrm{~b}$ ).

(4) Due to the previous step, the $\operatorname{acq}(\ell)$ event $e_{13}$ is now ordered before the rel $(\ell)$ event $e_{7}$. In order to not violate lock semantics, the critical section of the second process must be ordered before the first critical section of the first process. Hence we perform a lock-closure step, by ordering the rel $(\ell)$ event $e_{15}$ before the lock-acquire event $e_{4}$ (see dashed edge in Figure $2 \mathrm{~b}$ ).

(5) At this point, no other closure step is performed, and the partial order is called trace-closed. Note that there still exist conflicting accesses to variables $x_{1}$ and $x_{3}$ which are pairwise unordered and quite distant, hence not every linearization produces a valid trace, and a correct linearization is not obvious. We observe that we can obtain a valid trace by starting from the beginning of $\tau_{1}$ and $\tau_{2}$, and execute the former maximally and the latter minimally, according to the partial order. That is, we repeatedly execute $\tau_{1}$ until we reach an event that is preceded by an event of $\tau_{2}$, and then execute $\tau_{2}$ only until an event of $\tau_{1}$ becomes enabled again. This max-min linearization produces a valid witness trace (see Figure 2c).

In this work we make the above insights formal. We define the notion of trace-closed partial orders, which captures observation and lock-closure steps, and develop an efficient (polynomial-time) algorithm for computing the closure. For two processes, we show that max-min linearizations always produce valid traces, as long as the partial order is trace-closed. Hence, in this case, we have a sound and complete algorithm. The case of three or more processes is more complicated, and our algorithm might eventually order some (but crucially, not all) conflicting events arbitrarily. Although these choices might sacrifice completeness, the resulting partial orders are much weaker than before, so that complex races can still be exposed soundly by a max-min linearization.

\subsection{Our Contributions}

In summary, the contributions of this work are as follows. 
A new algorithm for dynamic race detection. Our main contribution is a polynomial-time and sound algorithm for detecting predictable races present in the input trace. In addition, our algorithm is complete for input traces that consist of events of two processes. First we study the decision problem, that is, given an input trace $t$ and a pair of events $\left(e_{1}, e_{2}\right)$ of $t$, decide whether the pair constitutes a data race of $t$. We present a sound algorithm for the problem that operates in $O\left(n^{2} \cdot \log n\right)$ time, where $n$ is the length of $t$. Since all data races can be computed by solving the decision problem for each of the $\left(\begin{array}{l}n \\ 2\end{array}\right)$ event pairs, we obtain a sound algorithm for reporting all races that requires $O\left(n^{4} \cdot \log n\right)$ time. In all cases, if the input trace consists of events of two processes, our race reports are also complete.

Our techniques rely on a new notion of trace-closed partial orders, which might be of independent interest. Informally, a closed partial order wrt a trace $t$ is a partial order over a subset of events of $t$ that respects (i) the observation $w(x)$ of each read event $r(x)$ in $t$, and (ii) the lock semantics. We define max-min linearizations of closed partial orders, and prove sufficient conditions under which a max-min linearization produces a valid trace. Finally, we show that given a partial order of small width, its closure can be computed in $O\left(n^{2} \cdot \log n\right)$ time. To this end, we develop a data structure DS for maintaining the incremental transitive closure of directed acyclic graphs of small width. DS requires $O(n)$ initialization time, after which it supports edge insertions and reachability queries in $O(\log n)$ time. Here, the width of partial orders is bounded by the number of processes, which is a small constant compared to the length of the trace, and hence our data structure is relevant.

A practical algorithm and implementation. We develop an algorithm for the function problem of race detection that is more practical than simply solving the decision problem for all possible pairs. The efficiency of the algorithm comes while retaining the soundness and completeness guarantees. We also develop sufficient conditions for detecting dynamically that our algorithm is complete for a given input, even in cases where completeness is not guaranteed theoretically.

We make a prototype implementation of our practical algorithm and evaluate it on a standard set of benchmark traces that contain hundreds of millions of events. We compare the performance of our tool against state-of-the-art, polynomial-time, partial-order-based methods, namely the HB [Lamport 1978], WCP [Kini et al. 2017], DC [Roemer et al. 2018] and SHB [Mathur et al. 2018] methods. Our approach detects significantly more races than each of these methods, while it has comparable running time, and typically being faster. In fact, our algorithm does not simply detect more races; it detects all races in the benchmark traces, and soundly reports that no more races (other than the detected ones) exist. To our knowledge, this is the first sound algorithm that achieves such a level of performance on both running time and completeness of the reported races.

\section{PRELIMINARIES}

In this section we introduce useful notation and define the problem of dynamic race detection for lock-based concurrent programs. The model follows similar recent works (e.g., [Kini et al. 2017]).

Concurrent program. Given a natural number $k$, let $[k]$ denote the set $\{1, \ldots, k\}$. We consider a shared-memory concurrent program $\mathcal{P}$ that consists of $k$ processes $\left\{p_{i}\right\}_{i \in[k]}$, under sequential consistency semantics. For simplicity of presentation we assume that $k$ is fixed a-priori, and no process is created dynamically. All results presented here can be extended to a setting with dynamic process creation. Communication between processes occurs over a set of global variables $\mathcal{G}$, and synchronization over a set of locks $\mathcal{L}$. We let $\mathcal{V}=\mathcal{G} \cup \mathcal{L}$ be the set of all variables of $\mathcal{P}$. Each process is deterministic, and performs a sequence of operations on execution. We are only interested in the operations that access a global variable or a lock, which are called events. 
(1) Given a global variable $x \in \mathcal{G}$, a process can write/read to $x$ via an event $w(x) / r(x)$.

(2) Given a lock $l \in \mathcal{L}$, a process can acquire $\ell$ via an event acq $(l)$ and release $l$ via an event rel $(l)$.

Each such event is atomic. Given an event $e$, we let loc(e) denote the global variable (or lock) that $e$ accesses. We denote by $\mathcal{W}_{p}$ (resp. $\mathcal{R}_{p}, \mathcal{L}_{p}^{A}, \mathcal{L}_{p}^{R}$ ) the set of all write (resp. read, acquire, release) events that can be performed by process $p$. We let $\mathcal{E}_{p}=W_{p} \cup \mathcal{R}_{p} \cup \mathcal{L}_{p}^{A} \cup \mathcal{L}_{p}^{R}$, and assume that $\mathcal{E}_{p} \cap \mathcal{E}_{p^{\prime}}=\emptyset$ for every $p \neq p^{\prime}$. We denote by $\mathcal{E}=\bigcup_{p} \mathcal{E}_{p}, \mathcal{W}=\bigcup_{p} \mathcal{W}_{p}, \mathcal{R}=\bigcup_{p} \mathcal{R}_{p}, \mathcal{L}^{A}=\bigcup_{p} \mathcal{L}_{p}^{A}$, $\mathcal{L}^{R}=\cup_{p} \mathcal{L}_{p}^{R}$ the events, write, read, acquire and release events of the program $\mathcal{P}$, respectively. Given an event $e \in \mathcal{E}$, we denote by $\mathrm{p}(e)$ the process that $e$ belongs to. Finally, given a set of events $X \subseteq \mathcal{E}$, we denote by $\mathcal{R}(X)$ (resp., $\left.\mathcal{W}(X), \mathcal{L}^{A}(X), \mathcal{L}^{R}(X)\right)$ the set of read (resp., write, lock-acquire, lock-release) events of $X$.

Conflicting events. Given two distinct events $e_{1}, e_{2} \in \mathcal{W} \cup \mathcal{R}$, we say that $e_{1}$ and $e_{2}$ are conflicting, denoted by $e_{1} \bowtie e_{2}$, if (i) $\operatorname{loc}\left(e_{1}\right)=\operatorname{loc}\left(e_{2}\right)$ (i.e., they access the same global variable) and (ii) $\left\{e_{1}, e_{2}\right\} \cap$ $\mathcal{W} \neq \emptyset$ (i.e., at least one is a write event). We extend the notion of conflict to locks, and say that two events $e_{1}, e_{2} \in \mathcal{L}^{A} \cup \mathcal{L}^{R}$ are conflicting if $\operatorname{loc}\left(e_{1}\right)=\operatorname{loc}\left(e_{2}\right)$ (i.e., they use the same lock).

Event sequences. Let $t$ be a sequence of events. We denote by $\mathcal{E}(t)$ the set of events, by $\mathcal{L}(t)$ the set of locks, and by $\mathcal{G}(t)$ the set of global variables in $t$. We let $\mathcal{W}(t)$ (resp., $\left.\mathcal{R}(t), \mathcal{L}^{A}(t), \mathcal{L}^{R}(t)\right)$ denote the set $\mathcal{W}(\mathcal{E}(t))$ (resp., $\left.\mathcal{R}(\mathcal{E}(t)), \mathcal{L}^{A}(\mathcal{E}(t)), \mathcal{L}^{R}(\mathcal{E}(t))\right)$. Given two distinct events $e_{1}, e_{2} \in \mathcal{E}(t)$, we say that $e_{1}$ is earlier than $e_{2}$ in $t$, denoted by $e_{1}<_{t} e_{2}$ iff $e_{1}$ appears before $e_{2}$ in $t$. We say that $e_{1}$ is program-ordered earlier than $e_{2}$, denoted by $e_{1}<\mathrm{PO}(t) e_{2}$, to mean that $e_{1}<_{t} e_{2}$ and $\mathrm{p}\left(e_{1}\right)=\mathrm{p}\left(e_{2}\right)$. When $t$ is clear from the context, we simply write $\mathrm{PO}$ to denote $\mathrm{PO}(t)$. We let $={ }^{t}$ be the identity relation on $\mathcal{E}(t)$, and denote by $\leq_{t}$, $\leq_{\mathrm{PO}}$ the relations $<_{t} \cup=_{t}$ and $<_{\mathrm{PO}} \cup=_{t}$ respectively. Given a set of events $X \subseteq \mathcal{E}$, we denote by $t \mid X$ the projection of $t$ onto $X$, i.e., it is the sub-sequence of events of $t$ that belong to $X$. Given two event sequences $t_{1}, t_{2}$, we denote by $t_{1} \circ t_{2}$ the concatenation of $t_{1}$ with $t_{2}$. Finally, given a process $p_{i}$, we let $t\left|p_{i}=t\right| \mathcal{E}_{p_{i}}$.

Lock events. Given a sequence of events $t$ and a lock-acquire event acq $\in \mathcal{L}^{A}(t)$, we denote by match $_{t}$ (acq) the earliest lock-release event in rel $\in \mathcal{L}^{R}(t)$ such that rel $\bowtie$ acq and acq $<_{t}$ rel, and let match $_{t}($ acq $)=\perp$ if no such lock-release event exists. If match $_{t}($ acq $) \neq \perp$, we require that $\mathrm{p}($ acq $)=\mathrm{p}\left(\operatorname{match}_{t}(\right.$ acq $\left.)\right)$, i.e., the two lock events belong to the same process. Similarly, given a

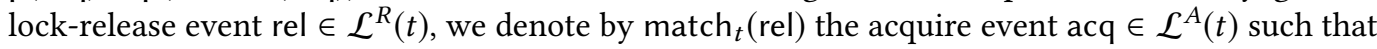
$\operatorname{match}_{t}(\mathrm{acq})=$ rel and require that such a lock-acquire event always exists.

Traces and observation functions. A sequence $t$ is called a trace if it satisfies the following.

(1) For every read event $r \in \mathcal{R}(t)$, there exists a write event $w \in \mathcal{W}(t) \operatorname{such}$ that $\operatorname{loc}(r)=\operatorname{loc}(w)$ and $w<_{t} r$.

(2) For any two lock-acquire events acq ${ }_{1}, \operatorname{acq}_{2} \in \mathcal{L}^{A}(t)$, if $\operatorname{loc}\left(\operatorname{acq}_{1}\right)=\operatorname{loc}\left(\operatorname{acq}_{2}\right)$ and acq $<_{t} \operatorname{acq}_{2}$, then $\operatorname{rel}_{1}=\operatorname{match}_{t}\left(\mathrm{acq}_{1}\right) \in \mathcal{L}^{R}(t)$ and $\operatorname{rel}_{1}<_{t} \mathrm{acq}_{2}$.

Given a trace $t$, we define its observation function $O_{t}: \mathcal{R}(t) \rightarrow \mathcal{W}(t)$ as follows: $O_{t}(r)=w$ iff

$$
\operatorname{loc}(r)=\operatorname{loc}(w) \quad \text { and } \quad w<_{t} r \quad \text { and } \quad \forall w^{\prime} \in \mathcal{W}(t) \backslash\{w\} \text { with } w \bowtie w^{\prime}: w^{\prime}<_{t} r \Rightarrow w^{\prime}<_{t} w
$$

In words, $O_{t}$ maps every read event $r$ to the write event $w$ that $r$ observes in $t$. For simplicity, we assume that $t$ starts with a write event to every location, hence $O_{t}$ is well-defined.

Enabled events and races. An event $e \in \mathcal{E}$ is said to be enabled in a trace $t$ if $t^{*}=t \circ e$ is a trace of $\mathcal{P}$. A trace $t$ is said to exhibit a race if there exist two consecutive conflicting events in $t$ that belong 
to different processes. Formally, there exist two events $e_{1}, e_{2} \in \mathcal{R} \cup \mathcal{W}$ such that (i) $\mathrm{p}\left(e_{1}\right) \neq \mathrm{p}\left(e_{2}\right)$, (ii) $e_{1} \bowtie e_{2}$, (iii) $e_{1}<_{t} e_{2}$, and (iv) for every $e \in \mathcal{E}(t) \backslash\left\{e_{1}, e_{2}\right\}$, we have that $e<_{t} e_{2} \Rightarrow e<_{t} e_{1}$.

Predictable races. A trace $t^{\prime}$ is a (prefix) correct reordering of another trace $t$ if (i) for every process $p_{i}$, we have that $t^{\prime} \mid p_{i}$ is a prefix of $t \mid p_{i}$ and (ii) $O_{t^{\prime}} \subseteq O_{t}$, i.e., the observation functions of $t^{\prime}$ and $t$ agree on their common read events. We say that $t$ has a predictable race on a pair of events $e_{1}, e_{2} \in \mathcal{E}(t)$ if there exists a correct reordering $t^{\prime}$ of $t$ such that $t^{*}=t^{\prime} \circ e_{1} \circ e_{2}$ is a trace that exhibits the race $\left(e_{1}, e_{2}\right)$.

Computational problems. The aim of this work is to present sound and fast algorithms for race detection, that also have certain completeness guarantees. As usual in algorithmic parlance, we are concerned with two versions of the problem, namely the following. Given an input trace $t$,

(1) the decision problem is stated on two events $e_{1}, e_{2} \in \mathcal{E}(t)$, and asks whether $\left(e_{1}, e_{2}\right)$ is a predictable race of $t$, and

(2) the function problem asks to compute the set of all pairs $\left\{\left(e_{1}^{i}, e_{2}^{i}\right)\right\}_{i}$ such that each $\left(e_{1}^{i}, e_{2}^{i}\right)$ is a predictable race of $t$.

Soundness, completeness and complexity. A predictive race-detection algorithm is called sound if on every input trace $t$, every reported race is a predictable race of $t$. The algorithm is called complete if it reports all predictable races of $t$. We note that these notions are often used in reverse in program verification. However, here we align with the terminology used in predictive techniques, hence soundness (resp., completeness) means the absence of false positives (resp., false negatives) We measure complexity in terms of the length $n$ of $t$. Other important parameters are the number of processes $k$ and the number of global variables $\mathcal{G}$. Typically $k$ is much smaller than $n$, and is treated as a constant. For simplicity, we also ignore $\mathcal{G}$ in our complexity statements. In all cases, our algorithms have a dependency of factor $k^{2} \cdot|\mathcal{G}|$ (and hence polynomial) on these parameters.

Dynamic process creation and other synchronization primitives. To keep the presentation simple, in the theoretical part of this work we neglect dynamic process creation (i.e., fork/join events). We note that such events can be handled naturally in our framework. In our experiments (Section 6) we explain how we handle dynamic process creation, which is present in our benchmark set. Similarly, our focus on locks is for simplicity of presentation and not restrictive to our model. For dynamic race detection, other synchronization primitives, such as compare-and-swap, intrinsic locks and synchronized methods can be simulated with locks and extra orderings in the partial orders. Indeed, this modeling approach has been taken in many other works, as e.g. in [Kini et al. 2017; Mathur et al. 2018; Roemer et al. 2018; Smaragdakis et al. 2012].

Due to limited space, all proofs are relegated to a technical report [Pavlogiannis 2019].

\section{TRACE-CLOSED PARTIAL ORDERS}

In this section we present relevant notation on partial orders, and introduce the concept of closed partial orders. We also present max-min linearizations which linearize closed partial orders to valid traces. Since this our most technical section, we provide here an overview to assist the reader.

(1) In Section 3.1 we define general notation on partial orders. Since these are partial orders over sets of events $X$ of an input trace $t$, we introduce a feasibility criterion for these sets, which requires that certain events are present in the partial order. For example, for every two conflicting lock-acquire events in $X$, at least one corresponding lock-release event must also be in $X$.

(2) In Section 3.2 we define trace-closed partial orders. Intuitively, this notion requires certain orderings between conflicting events to be present in the partial order. Note that not every 
linearization of a partial order leads to a valid trace (e.g., some linearizations might not respect the lock semantics). Nevertheless, we show that for a specific class of trace-closed partial orders, a specific type of max-min linearization is guaranteed to always produce a valid trace.

(3) In Section 3.3 we develop an algorithm that computes the trace-closure of a partial order efficiently. To this end, we develop a data structure DS for the efficient representation of partial orders. For ease of presentation, we relegate the technical description of DS in Appendix A.

\subsection{Partial Orders}

Feasible sets. Given a set of events $X \subseteq \mathcal{E}(t)$, we say that $X$ is prefix-closed for $t$ if for every pair of events $e_{1}, e_{2} \in \mathcal{E}(t)$ if $e_{1} \leq \mathrm{PO} e_{2}$ and $e_{2} \in X$, then $e_{1} \in X$ (i.e., $X$ is an ideal of $\leq_{\mathrm{PO}}$ ). We define the open acquires of $X$ under $t$ as $\operatorname{OpenAcqs}_{t}(X)=\left\{\right.$ acq $\in \mathcal{L}^{A}(X): \operatorname{match}_{t}($ acq $\left.) \notin X\right\}$.

We call $X$ observation-feasible for $t$ if for every read event $r \in \mathcal{R}(X)$, we have $O_{t}(r) \in X$. We call $X$ lock-feasible for $t$ if (i) for every lock-release event rel $\in \mathcal{L}^{R}(X)$, we have match $_{t}($ rel $) \in X$, and (ii) for every distinct pair of lock-acquire events acq ${ }_{1}, \operatorname{acq}_{2} \in \operatorname{OpenAcqs}_{t}(X)$, we have $\operatorname{loc}\left(\operatorname{acq}_{1}\right) \neq$ $\operatorname{loc}\left(\mathrm{acq}_{2}\right)$. In words, $X$ is lock-feasible if every release event of $X$ has its matching acquire event also in $X$, and every open lock of $X$ remains open by exactly one acquire event of $X$. Finally, we call $X$ feasible for $t$ if $X$ is prefix-closed, observation-feasible, and lock-feasible for $t$.

Partial orders. Given a trace $t$ and a set $X \subseteq \mathcal{E}(t)$, a partial order $P(X)$ over $X$ is a reflexive, antisymmetric and transitive relation over $X$ (i.e., $\left.\leq_{P(X)} \subseteq X \times X\right)$. When $X$ is clear from the context, we will simply write $P$ instead of $P(X)$. Given two events $e_{1}, e_{2}$ we write $e_{1}<_{P} e_{2}$ to denote that $e_{1} \leq_{P} e_{2}$ and $e_{1} \neq e_{2}$. Given two distinct events $e_{1}, e_{2} \in X$, we say that $e_{1}$ and $e_{2}$ are unordered by $P$, denoted by $e_{1} \|_{P} e_{2}$, if neither $e_{1}<_{P} e_{2}$ nor $e_{2}<_{P} e_{1}$. Given a set $Y \subseteq X$, we denote by $P \mid Y$ the projection of $P$ on $Y$, i.e., we have $\leq_{P \mid Y} \subseteq Y \times Y$, and for all $e_{1}, e_{2} \in Y, e_{1} \leq_{P \mid Y} e_{2}$ iff $e_{1} \leq_{P} e_{2}$. Given two partial orders $P$ and $Q$ over a common set $X$, we say that $Q$ refines $P$, denoted by $Q \sqsubseteq P$, if for every pair of events $e_{1}, e_{2} \in X$, if $e_{1} \leq_{P} e_{2}$ then $e_{1} \leq_{Q} e_{2}$. If $Q$ refines $P$, we say that $P$ is weaker than $Q$. A linearization of $P$ is a total order that refines $P$. We make the following remark.

Remark 1. Not every linearization of a partial order $P$ is a valid trace, and generally, $P$ is not guaranteed to have such a linearization. Our algorithm for dynamic race detection relies on developing sufficient conditions under which $P$ indeed has a linearization to a valid trace.

Width and Mazurkiewicz traces. Let $P$ be a partial order over a set $X \subseteq \mathcal{E}(t)$. The width width $(P)$ of $P$ is the length of its longest antichain. i.e., it is the largest size of a set $Y \subseteq X$ such that for every pair of distinct elements $e_{1}, e_{2} \in Y$ we have $e_{1} \|_{P} e_{2}$. The partial order $P$ is called a Mazurkiewicz trace (or $M$-trace for short) if for every two conflicting events $e_{1}, e_{2} \in X$, we have $e_{1} \nVdash_{P} e_{2}$ [Mazurkiewicz 1987]. Note that if width $(P)=1$ then $P$ is trivially an M-trace.

\subsection{Trace-closed Partial Orders}

In this section we define the notion of trace-closed partial orders. This is a central concept in this work, as our race-detection algorithm is based on computing trace-closed partial orders efficiently.

Trace-respecting partial orders. Let $t$ be a trace, and $P$ a partial order over a feasible set $X \subseteq \mathcal{E}(t)$. We say that $P$ respects $t$ if the following conditions hold.

(1) $P \sqsubseteq \mathrm{PO} \mid X$, i.e., $P$ refines the program order when restricted to the set $X$.

(2) For every read event $r \in \mathcal{R}(X)$ we have $O_{t}(r)<_{P} r$.

(3) For every lock-acquire event acq $\in \mathcal{L}^{A}(X)$, if match $_{t}$ (acq) $\notin X$, then for every lock-release event rel $\in \mathcal{L}^{R}(X)$ such that rel $\bowtie$ acq, we have rel $<_{P}$ acq. 
We denote by $R_{t}(X)$ the weakest partial order over $X$ that respects $t$.

Trace-closed partial orders. Let $t$ be a trace, and $P$ a partial order over a feasible set $X \subseteq \mathcal{E}(t)$ such that $P$ respects $t$. We call $P$ observation-closed if the following condition holds. For every read event $r \in \mathcal{R}(X)$, let $w=O_{t}(r)$. For every write event $w^{\prime} \in \mathcal{W}(X) \backslash\{w\}$ such that $w^{\prime} \bowtie r$, we have

$$
\text { if } w^{\prime}<_{P} r \text { then } w^{\prime}<_{P} w \text { and if } w<_{P} w^{\prime} \text { then } r<_{P} w^{\prime}
$$

For a pair of lock-release events rel ${ }_{1}, \operatorname{rel}_{2} \in \mathcal{L}^{R}(X)$, let acq $q_{i}=$ match $_{t}\left(\right.$ rel $\left._{i}\right)$. We call $P$ lock-closed if

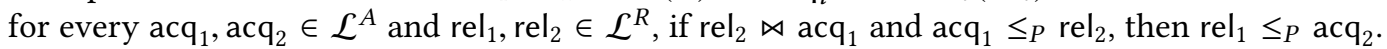
Finally, we call $P$ trace-closed (or simply closed) if it is both observation-closed and lock-closed. See Figure 3 for an illustration. Note that a closed partial order can still contain conflicting events that are unordered. In addition, it does not necessarily admit a linearization to a valid trace. In the next paragraph we develop sufficient conditions for when such a linearization exists.

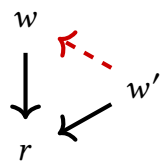

(a)

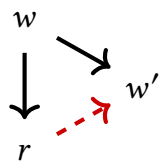

(b)

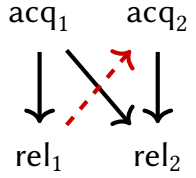

(c)

Fig. 3. The conditions of observation closure $(a, b)$ and lock closure (c). Solid edges and dashed edges represent existing and inferred orderings, respectively.

Max-min linearizations. The key technical challenge in race prediction is, given a trace $t$, to construct a partial order $P$ over $\mathcal{E}(t)$ such that $P$ is efficiently linearizable to a correct reordering of $t$ that manifests the race. Here we use trace-closed partial orders to provide a sufficient condition for efficient linearization, which we call the max-min linearization. In later sections, our race-detection algorithm constructs trace-closed partial orders. The max-min linearization of such partial orders will guarantee that the races exposed by these partial orders are indeed valid races, which are exhibited by a trace constructed using the max-min linearization.

Let $t$ be a trace, and consider a partial order $P$ over a feasible set $X \subseteq \mathcal{E}(t)$ such that $P$ is trace-closed for $t$ and $X$ can be partitioned into two sets $X_{1}, X_{2} \subseteq X$ such that (i) width $\left(P \mid X_{1}\right)=1$ and (ii) $P \mid X_{2}$ is an M-trace. The max-min linearization $t^{*}$ is a linearization of $P$ given by Algorithm 1 . In words, first every event of $X_{1}$ is ordered before every event of $X_{2}$, as long as this is allowed by $P$, and then the resulting partial order is linearized arbitrarily. Intuitively, we obtain the sequence $t^{*}$ by linearizing $X_{1}$ maximally, and $X_{2}$ minimally. See Figure 4 for an illustration.

Intuition. First, observe that $P$ can contain pairs of conflicting events that are unordered, i,.e., between the sets $X_{1}$ and $X_{2}$. Conceptually, MaxMin shows that as we attempt to linearize $P$, we do not have to make an exhaustive search over all the possible (exponentially many) orderings of such pairs. Instead, the specific orderings made by MaxMin are guaranteed to produce a correct linearization. The intuition behind the correctness of MaxMin can be summarized as follows.

(1) Since width $\left(P \mid X_{1}\right)=1$, ordering every two events $e_{1} \in X_{1}, e_{2} \in X_{2}$ as $e_{1} \rightarrow e_{2}$ (provided that $e_{2} \nless_{P} e_{1}$ ) creates a partial order (i.e., no cycle is formed).

(2) Since $P$ is closed and $P \mid X_{2}$ is an M-trace, this ordering respects the observation $w$ of every read event $r$. Indeed, if the ordering was forcing some other conflicting write event $w^{\prime}$ between $w$ and $r$, then $w^{\prime}$ must be ordered with at least one of $w$ and $r$, and then the corresponding closure rule (Figure 3) would have resolved this conflict entirely. 
Theorem 3.1. Let $t$ be a trace and $P$ a partial order over a feasible set $X \subseteq \mathcal{E}(t)$ such that $P$ is traceclosed for $t$ and $X$ can be partitioned into two sets $X_{1}, X_{2}$ so that (i) width $\left(P \mid X_{1}\right)=1$ and (ii) $P \mid X_{2}$ is a Mazurkiewicz trace. The max-min linearization of $P$ produces a correct reordering of $t$.

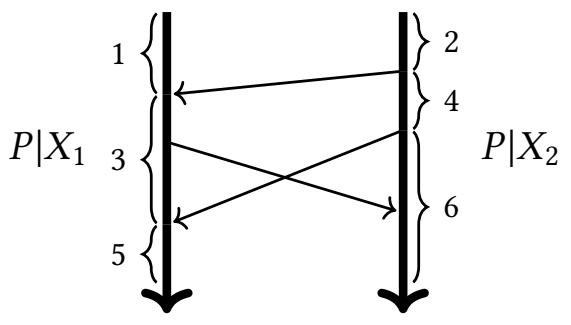

Fig. 4. Illustration of the max-min linearization. Here width $\left(P \mid X_{1}\right)=$ width $\left(P \mid X_{2}\right)=1$. The numbers show the order in which various segments of the sets $X_{1}$ and $X_{2}$ are executed, i.e., the linearization $t^{*}$ consists of the segments $t^{*}=\langle 1\rangle \circ\langle 2\rangle \circ\langle 3\rangle \circ\langle 4\rangle \circ\langle 5\rangle \circ\langle 6\rangle$. Theorem 3.1 guarantees that $t^{*}$ is a correct reordering.

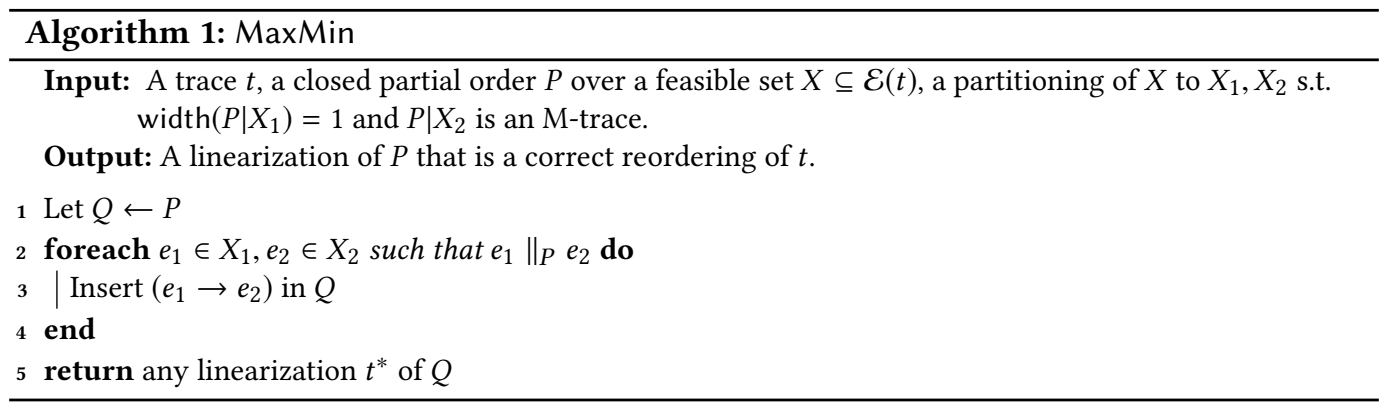

\subsection{Computing the Closure of a Partial Order}

In this section we define the trace-closure of partial orders, and develop an efficient algorithm that, given a partial order $P$, either computes the closure of $P$ or concludes that the closure does not exist. In the next section we will solve the decision problem of race detection by constructing specific partial orders and computing their closure.

Feasible partial orders. Let $t$ be a trace and $P$ a partial order over a feasible set $X \subseteq \mathcal{E}(t)$ such that $P$ respects $t$. If there exists a partial order $Q$ over $X$ such that (i) $Q \sqsubseteq P$ and (ii) $Q$ is closed, we define the closure of $P$ as the smallest such partial order $Q$. If no such partial order $Q$ exists, then the closure of $P$ is undefined (i.e., $P$ does not have a closure). We call $P$ feasible iff it has a closure. The following lemma states that $P$ has a unique closure.

Lemma 3.2. There exists at most one smallest partial order $Q$ such that (i) $Q \sqsubseteq P$ and (ii) $Q$ is closed.

Computing the closure of a partial order. It is straightforward to verify that, given a partial order $P$, the closure of $P$ (or deducing that $P$ is not feasible) can be computed in polynomial time. This is simply achieved by iteratively detecting whether one of the cases shown in Figure 3 is violated, and strengthening $P$ with the appropriate orderings. However, since our goal is to handle large traces with hundreds of millions of events, polynomial-time guarantees are not enough, and 
the goal is to develop an algorithm with low polynomial complexity. Here we develop such an algorithm called, Closure, that computes the closure of a partial order in $O\left(n^{2} \cdot \log n\right)$ time.

The data structure DS. To make the closure computation efficient, we develop a data structure DS for manipulating partial-orders efficiently. Given a partial order $P$ over $n$ events such that $P$ has width $k=O(1)$, DS represents $P$ in $O(n)$ space and supports the following operations: (i) initialization in $O(n)$ time, (ii) querying whether $e_{1} \leq_{P} e_{2}$, for any two events $e_{1}, e_{2}$ in $O(\log n)$ time, and (iii) inserting an ordering $e_{1} \leq_{P} e_{2}$, for any two events $e_{1}, e_{2}$ in $O(\log n)$ time. For ease of presentation, we relegate the formal description of DS to Appendix A.

The event maps After, Before and $\mathcal{F}$. Consider a trace $t$. For every lock $l \in \mathcal{L}(t)$, we define the maps After ${ }_{l}^{\mathcal{L}^{A}}$, After $_{l}^{\mathcal{L}^{R}}$, Before ${ }_{l}^{\mathcal{L}^{A}}$, Before ${ }_{l}^{\mathcal{L}^{R}}: \mathcal{E}(t) \rightarrow \mathcal{E}(t) \cup\{\perp\}$, as follows. Given an event $e \in \mathcal{E}(t)$, the maps $\operatorname{After}_{l}^{\mathcal{L}^{A}}(e)$ and $\operatorname{Before}_{l}^{\mathcal{L}^{A}}(e)$ point to the first lock-acquire event acq after $e$ in $t$, and last lock-acquire event acq before $e$ in $t$, respectively, such that $\mathrm{p}(e)=\mathrm{p}($ acq $)$ and $\operatorname{loc}($ acq $)=l$. The maps $\operatorname{After}_{l}^{\mathcal{L}^{R}}(e)$ and $\operatorname{Before}_{l}^{\mathcal{L}^{R}}(e)$ are defined analogously, pointing to lockrelease instead of lock-acquire events. Similarly, for every global variable $x \in \mathcal{G}(t)$, we define the maps After ${ }_{x}^{\mathcal{W}}$, After $_{x}^{\mathcal{R}}$, Before $_{x}^{\mathcal{W}}$, Before $_{x}^{\mathcal{R}}: \mathcal{E}(t) \rightarrow \mathcal{E}(t) \cup\{\perp\}$, as follows. Given an event $e \in \mathcal{E}(t)$ the map $\operatorname{After}_{x}^{\mathcal{W}}(e)$ (resp. Before $\left.x_{x}^{\mathcal{W}}(e)\right)$ points to the first write event $w$ after (resp., before) $e$ in $t$ such that $\mathrm{p}(e)=\mathrm{p}(w)$ and $\operatorname{loc}(w)=x$. The maps $\operatorname{After}_{l}^{\mathcal{R}}(e)$ and $\operatorname{Before}_{l}^{\mathcal{R}}(e)$ are defined analogously, pointing to read instead of write events. Finally, the flow map $\mathcal{F}_{p}: \mathcal{W}(t) \rightarrow \mathcal{R}(t) \cap \mathcal{R}_{p}$ of $t$ is a partial function that maps each write event $w$ to the last read event of $p$ that observes $w$. In all the above cases, if no corresponding event exists, the respective map points to $\perp$. Observe that each of these maps has size $O(|G| \cdot n)$, where $|G|$ is the number of memory locations of $t$. The maps can be constructed in $O(|G| \cdot n)$ time, simply by traversing $t$ and maintaining on-the-fly each map.

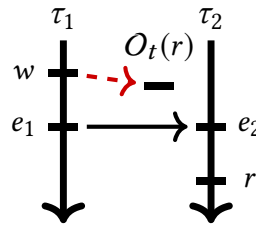

(a)

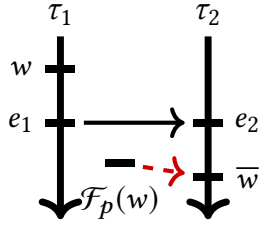

(b)

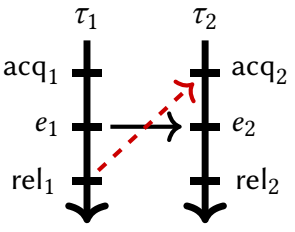

(c)

Fig. 5. Illustration of ObsClosure $\left(e_{1}, e_{2}\right)(\mathrm{a}, \mathrm{b})$ and LockClosure $\left(e_{1}, e_{2}\right)(\mathrm{c})$ for an edge $\left(e_{1}, e_{2}\right)$ added in $P$. In each case, the dashed edge corresponds to the new ordering inserted in $P$. Recall that $O_{t}(r)$ denotes the observation of $r$ in $t$, and $\mathcal{F}_{p}(w)$ denotes the last read event of process $p$ that observes $w$ in $t$.
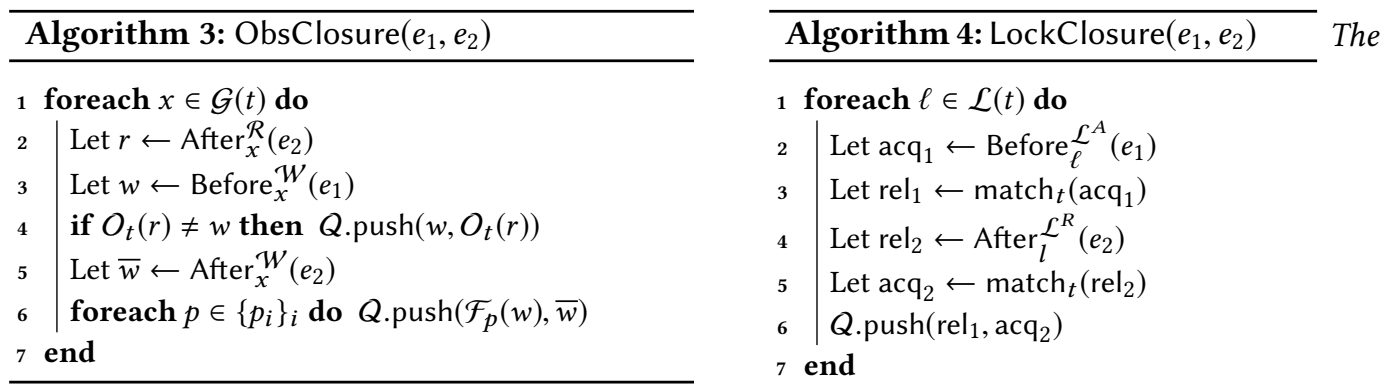

algorithm Closure. We now present Closure for computing the closure of a partial order $P$ over a set $X$, or concluding that $P$ is not feasible. The algorithm maintains a partial order as a DAG 


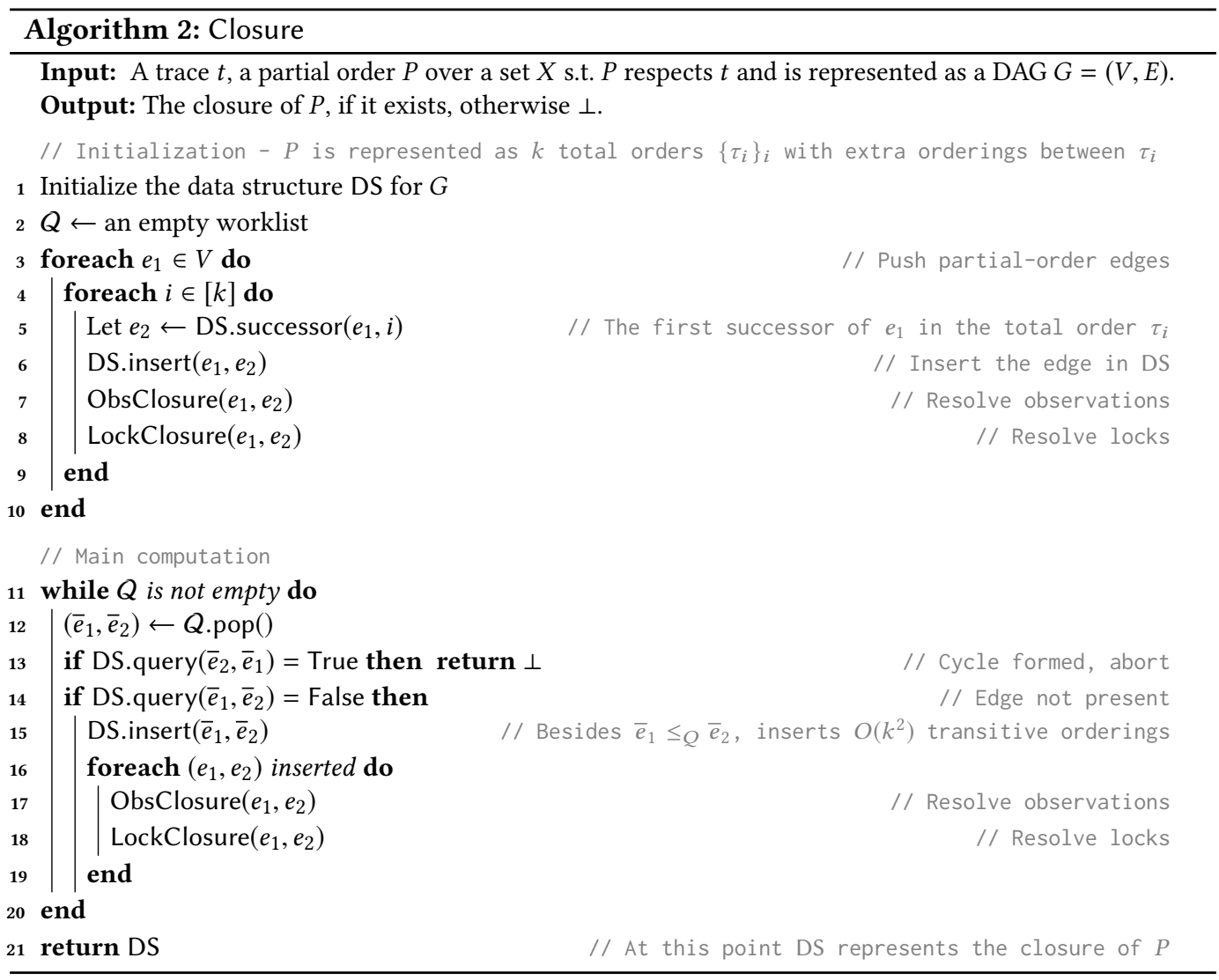

represented by the data structure DS. Conceptually, DS consists of $k$ total orders, $\tau_{1}, \ldots, \tau_{k}$, where $k=$ width $(P)$, with some extra orderings that go across the $\tau_{i}$. Each total order $\tau_{i}$ contains the events of process $p_{i}$ in $X$. Initially DS represents $P$. The main computation iterates over a worklist $Q$ which holds edges to be inserted in DS. Upon extracting such an edge $\left(\bar{e}_{1}, \bar{e}_{2}\right)$ from $Q$, the algorithm inserts the edge in DS using the operation DS.insert. This operation results in various edges $\left(e_{1}, e_{2}\right)$ inserted in the graph, transitively through $\left(\bar{e}_{1}, \bar{e}_{2}\right)$. For every $\left(e_{1}, e_{2}\right)$, the algorithm calls methods ObsClosure and LockClosure to resolve any violation of observation and lock constraints created by the insertion of $\left(e_{1}, e_{2}\right)$. Figure 5 illustrates ObsClosure and LockClosure. Algorithm 2, Algorithm 3 and Algorithm 4 give the description of Closure, ObsClosure and LockClosure, respectively.

Correctness and complexity. It is rather straightforward that if $P$ has a closure $Q$, then for each $Q$.push $\left(e_{1}, e_{2}\right)$ operation performed by Closure, we have $e_{1}<_{Q} e_{2}$. It follows that if Closure returns $\perp$, then $P$ is unfeasible. On the other hand, if Closure does not return $\perp$, then the partial order $Q$ stored in the data structure DS is the closure of $P$. Indeed, each of the closure rules can only be violated by an ordering $e_{1}<_{Q} e_{2}$. The algorithm guarantees that every such edge is processed by the methods ObsClosure and LockClosure, and new edges will be inserted in DS according to the rules of Figure 5. After such edges have been inserted, the ordering $e_{1}<_{Q} e_{2}$ can no longer violate any of the conditions of closure. Regarding the time complexity, the algorithm inserts at most $n^{2}$ edges in the partial order represented by DS. Using the algorithms for DS (see Lemma A.1 in 
Appendix A), for every edge inserted by the algorithm, identifying which other edges are imposed by the closure rules requires only $O(\log n)$ time. We have the following theorem.

Theorem 3.3. Let $t$ be a trace and $P$ a partial order over a feasible set $X \subseteq \mathcal{E}(t)$ such that $P$ respects $t$. Closure correctly computes the closure of $P$ and requires $O\left(n^{2} \cdot \log n\right)$ time.

Incremental closure. In our race detection algorithm, we also make use of the following operation on partial orders. Let $t$ be a trace and $P$ a partial order over a feasible set $X \subseteq \mathcal{E}(t)$ and such that $P$ is closed wrt $t$ and is represented as a DAG using the data structure DS. Given a pair of events $e_{1}, e_{2} \in X$, we define the operation InsertAndClose $\left(P, e_{1} \rightarrow e_{2}\right)$ as follows. We execute the algorithm Closure starting from Line 15, performing a DS.insert $\left(e_{1}, e_{2}\right)$. Hence we perform the ObsClosure and LockClosure only for the new orderings added due to $\left(e_{1}, e_{2}\right)$.

Lemma 3.4. Let $\Sigma$ be a sequence of InsertAndClose operations. Performing all operations of $\sum$ requires $O\left(n^{2} \cdot \log n+|\Sigma| \cdot \log n\right)$ time in total, and produces a closed partial order.

\section{THE DECISION PROBLEM OF RACE DETECTION}

Here we present a polynomial-time algorithm for the decision problem of dynamic race detection, i.e., given an input trace $t$ and two events $e_{1}, e_{2} \in \mathcal{E}(t)$, decide whether $\left(e_{1}, e_{2}\right)$ is a predictable race of $t$. Our algorithm is sound but incomplete in general, and it becomes complete if the input trace contains events of only two processes. To assist the reader, we provide an outline of this section.

(1) First, we introduce the notion of relative causal cone of an event $e$, which is a subset of events of $t$. Intuitively, it can be thought of as a trace slice of $t$ up to $e$. When deciding whether $\left(e_{1}, e_{2}\right)$ is a race of $t$, our algorithm tries to find a witness trace for the race, such that the witness consists of events of the causal cones of $e_{1}$ and $e_{2}$.

(2) Second, we present our main algorithm RaceDecision for deciding whether $\left(e_{1}, e_{2}\right)$ is a predictable race of $t$. In high level, the algorithm computes the causal cones of $e_{1}$ and $e_{2}$, and constructs a partial order $P$ over events of the causal cones. Afterwards, it computes the closure $Q$ of $P$ using algorithm Closure from the previous section, and resolves certain orderings of conflicting events in $Q$ according to the trace order in $t$. Finally, it uses algorithm MaxMin to linearize $Q$ to a witness trace that exhibits the race. We also prove that this process is sound, i.e., if $\left(e_{1}, e_{2}\right)$ is reported as a race, then it is a true predictable race of $t$.

(3) Third, we prove that the above process is also complete for traces that consist of events of two processes, i.e., it detects all predictable races.

(4) Finally, we illustrate RaceDecision on a few examples.

Relative causal cones. Given a trace $t$, an event $e \in \mathcal{E}(t)$ and a process $p$, the causal past cone RCone $_{t}(e, p)$ of $e$ relative to $p$ in $t$ is the smallest set that contains the following events:

(1) For every event $e^{\prime} \in \mathcal{E}(t)$ with $e^{\prime}<\mathrm{pO} e$, we have that $e^{\prime} \in \mathrm{RCone}_{t}(e, p)$.

(2) For every pair of events $e_{1} \in \mathrm{RCone}_{t}(e, p)$ and $e_{2} \in \mathcal{E}(t)$, if $e_{2} \leq_{\mathrm{PO}} e_{1}$ then $e_{2} \in \mathrm{RCone}_{t}(e, p)$.

(3) For every read event $r \in \mathcal{R}\left(\mathrm{RCone}_{t}(e, p)\right)$, we have that $\mathrm{O}_{t}(r) \in \mathrm{RCone}_{t}(e, p)$.

(4) For every lock-acquire event acq $\in \operatorname{RCone}_{t}(e, p)$, if $\mathrm{p}($ acq $) \neq \mathrm{p}(e)$ and $\mathrm{p}($ acq $) \neq p$, then $\operatorname{match}_{t}($ acq $) \in$ RCone $_{t}(e, p)$.

It is easy to verify that $\mathrm{RCone}_{t}(e, p)$ is always observation-feasible but not necessarily lock-feasible.

Intuition and example on relative causal cones. In order to decide whether an event pair $\left(e_{1}, e_{2}\right)$ is a predictable race of an input trace $t$, we first need to decide the events that will constitute 
a witness trace $t^{*}$ that exposes the race. In our race-detection algorithm, we take this set to be RCone $_{t}\left(e_{1}, \mathrm{p}\left(e_{2}\right)\right) \cup \mathrm{RCone}_{t}\left(e_{2}, \mathrm{p}\left(e_{1}\right)\right)$, i.e., it is the causal past cone of each focal event relative to the process of the other focal event. Conditions 1-3 ensure that the cones are closed wrt the program order, and the observation of every read event is present, which is required for $t^{*}$ to be a correct reordering of $t$. The intuition behind condition 4 is a bit more subtle. To avoid having two critical sections on the same lock open, we include the matching release event of every lock-acquire event. However, this rule does not apply for the processes of the focal events, since for these processes the events we have to include in $t^{*}$ are precisely the predecessors of the corresponding focal event.

Consider the input trace in Figure 6, where our task is to detect the race $\left(e_{2}, e_{10}\right)$. We outline here the computation of the relative causal cones RCone $t_{t}\left(e_{1}, \mathrm{p}\left(e_{2}\right)\right)$ and RCone $e_{t}\left(e_{2}, \mathrm{p}\left(e_{1}\right)\right)$. Item 1 of relative causal cones leads to RCone ${ }_{t}\left(e_{2}, p_{3}\right)=\left\{e_{1}\right\}$. For RCone $t_{t}\left(e_{10}, p_{1}\right)$, Item 1 makes $e_{9} \in \mathrm{RCone}_{t}\left(e_{10}, p_{1}\right)$. Since $e_{9}$ is a read event, Item 3 makes $O_{t}\left(e_{9}\right)=e_{5} \in \mathrm{RCone}_{t}\left(e_{10}, p_{1}\right)$, and then Item 2 makes $e_{4} \in$ RCone $_{t}\left(e_{10}, p_{1}\right)$. Since $e_{4}$ is a lock-acquire event and $\mathrm{p}\left(e_{4}\right) \neq p_{1}, p_{3}$ (i.e., the process of $e_{4}$ is neither the process of $e_{10}$, nor the process relative to which we are computing the causal cone of $e_{10}$ ), Item 4 makes match $_{t}\left(e_{4}\right)=e_{6} \in \mathrm{RCone}_{t}\left(e_{10}, p_{1}\right)$. Hence, in the end, RCone ${ }_{t}\left(e_{10}, p_{1}\right)=\left\{e_{9}, e_{6}, e_{5}, e_{4}\right\}$.

\begin{tabular}{r|l|l|l|} 
& $\tau_{1}$ & $\tau_{2}$ & $\tau_{3}$ \\
\hline 1 & $\operatorname{acq}(\ell)$ & & \\
2 & $\mathbf{w}(\mathbf{x})$ & & \\
3 & $\operatorname{rel}(\ell)$ & & \\
4 & & $\operatorname{acq}(\ell)$ & \\
5 & & $w(y)$ & \\
6 & & $\operatorname{rel}(\ell)$ & \\
7 & $w(z)$ & & \\
8 & & $r(z)$ & \\
9 & & & $r(y)$ \\
10 & & & $\mathbf{w}(\mathbf{x})$ \\
\hline
\end{tabular}

(a) An input trace $t$.

\author{
RCone $_{t}\left(e_{2}, p_{3}\right)=\left\{e_{1}\right\}$ \\ RCone $_{t}\left(e_{10}, p_{1}\right)=\left\{e_{9}, e_{6}, e_{5}, e_{4}\right\}$
}

(b) The relative causal cones RCone ${ }_{t}\left(e_{2}, p_{3}\right)$ and $\mathrm{RCone}_{t}\left(e_{10}, p_{1}\right)$

Fig. 6. The relative causal cones when testing for a race $\left(e_{2}, e_{10}\right)$.

The algorithm RaceDecision. We now describe our algorithm for reporting whether $t$ has a predictable race on a given pair $\left(e_{1}, e_{2}\right)$. In words, the algorithm constructs a set $X$ that is the union of the causal cones of each $e_{i}$ relative to the process of $e_{3-i}$. Afterwards, the algorithm constructs a partial order $P$ that respects $X$, and computes the closure $Q$ of $P$. Finally, the algorithm non-deterministically chooses some $i \in[2]$, and examines all events that belong to processes other than $p_{i}$. For every two such events $\bar{e}_{1}, \bar{e}_{2}$, if they conflict and are unordered by $Q$, the algorithm orders them according to their order in $t$. If a cycle is created in $Q$ during this process, the algorithm returns False. Otherwise, at the end of this process, the set $X$ can be naturally partitioned into two sets $X_{1}, X_{2}$ such that width $\left(Q \mid X_{1}\right)=1$ and $Q \mid X_{2}$ is an M-trace. The first set is $X_{1} \mid \mathrm{p}\left(e_{i}\right)$, i.e., it contains the events of the process in which $e_{i}$ belongs to, and thus is a totally ordered set under $Q$. The second set is $X_{2}=X \backslash X_{1}$, and note that all pairs of conflicting events of $X_{2}$ are now ordered under $Q$. Hence, according to Theorem 3.1, the partial order $Q$ is linearizable to a valid trace, and the algorithm returns True. See Algorithm 5 for a formal description.

The complexity of the algorithm is $O\left(n^{2} \cdot \log n\right)$, which is the time required for computing the closure of the partial order $Q$ in Line 4 and Line 8 (due to Theorem 3.3 and Lemma 3.4, respectively). 
Note that the algorithm is sound, i.e., if it returns True then $\left(e_{1}, e_{2}\right)$ is a true predictable race of $t$. On the other hand, the algorithm is incomplete in general, i.e., it might return False even though $\left(e_{1}, e_{2}\right)$ is a true predictable race of $t$. For example, in Line 9, the algorithm orders some pairs of conflicting events $\left(\bar{e}_{1}, \bar{e}_{2}\right)$ in the same order as in the input trace. Although these orderings are expected to work most of the time, this choice might not always be correct. In such cases, a cycle will be created in $Q$, and the algorithm will return False (see [Pavlogiannis 2019] for an example). As we discuss next, the algorithm becomes complete if the input trace consists of only two processes.

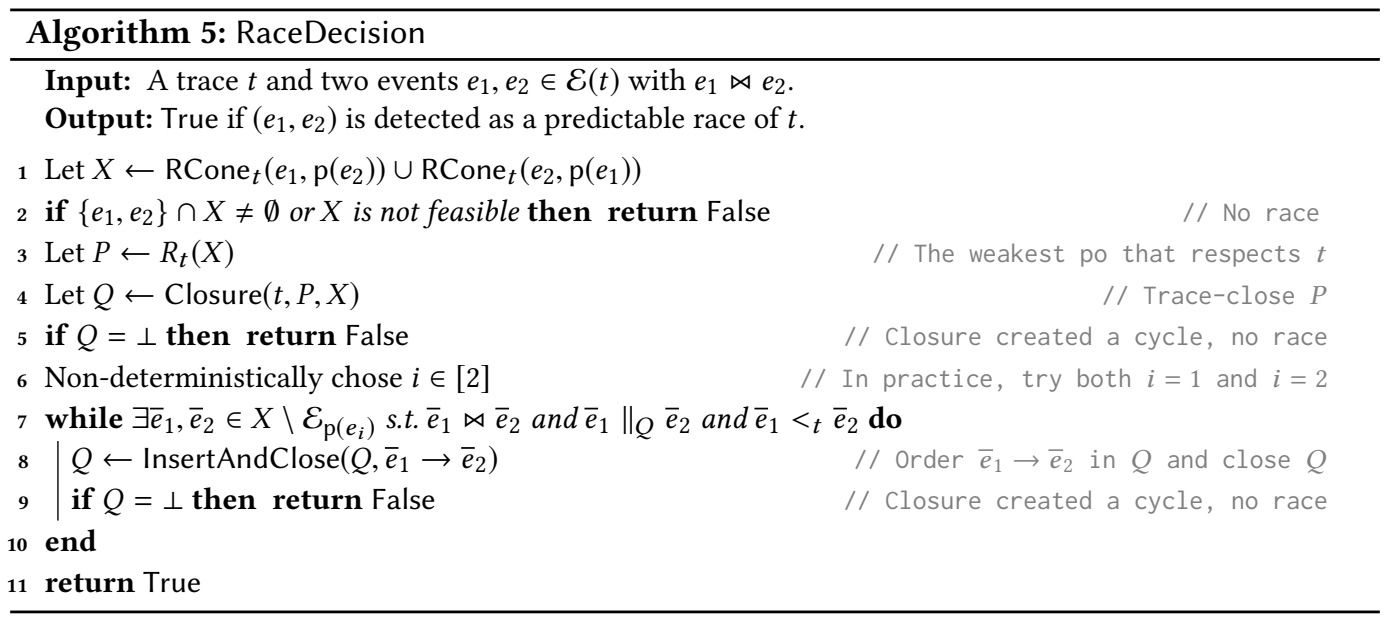

Completeness for two processes. We now discuss the completeness properties of RaceDecision for reporting races on input traces of two processes. Assume that $\left(e_{1}, e_{2}\right)$ is a race of the input trace. The key insight is that Line 7 of RaceDecision is not executed, as every pair of events $\bar{e}_{1}, \bar{e}_{2}$ in that line belong to the same process, and thus are already ordered. Up until that point, all orderings used in constructing the partial order $R_{t}(X)$ and computing the closure of $R_{t}(X)$ are necessarily present in every trace that witnesses the race $\left(e_{1}, e_{2}\right)$. Hence the closure computation cannot return $\perp$, and RaceDecision returns True. The following theorem concludes the results of this section.

Theorem 4.1. Let $t$ be a trace of $k \geq 2$ processes, and $n=|\mathcal{E}(t)|$. Let $e_{1}, e_{2} \in \mathcal{E}(t)$ be two conflicting events of $t$. The algorithm RaceDecision requires $O\left(n^{2} \cdot \log n\right)$ time and soundly reports whether $\left(e_{1}, e_{2}\right)$ is a predictable race of $t$. If $k=2$, RaceDecision is also complete. If RaceDecision reports a race, a witness trace can be constructed in $O(n \cdot \log n)$ time.

As there are $O\left(n^{2}\right)$ pairs of events in $t$, Theorem 4.1 yields the following corollary.

COROLLARY 4.2. Let $t$ be a trace of $k \geq 2$ processes. There exists a sound algorithm that requires $O\left(n^{4} \cdot \log n\right)$ time and soundly reports predictable races of $t$. If $k=2$, the algorithm is also complete (i.e., it reports all predictable races).

Remark 2. We note that the dependency of Corollary 4.2 on the number of variables $|\mathcal{G}|$ and number of threads $k$ is $O\left(|\mathcal{G}| \cdot k^{2} \cdot n^{4} \cdot \log n\right)$. To keep the presentation simple, we have neglected the dependency on $|\mathcal{G}|$ and $k$ in the analysis of the algorithm. 


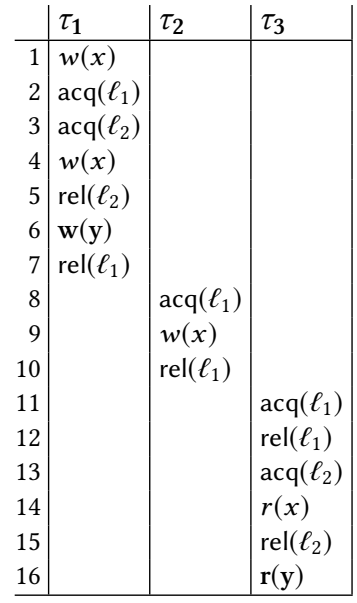

(a) Is $\left(e_{6}, e_{16}\right)$ a race?

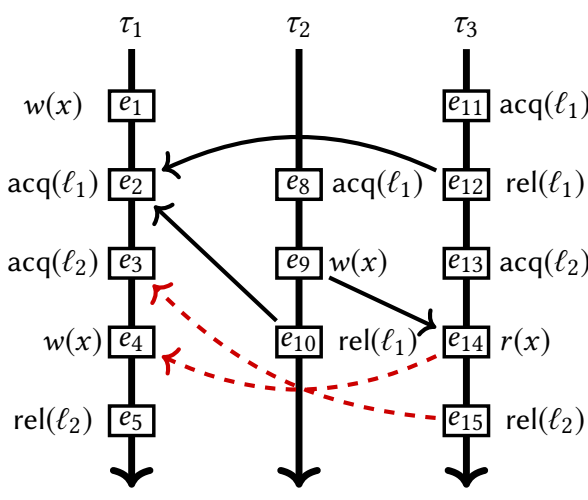

(b) The partial order $P$ and its closure $Q$.

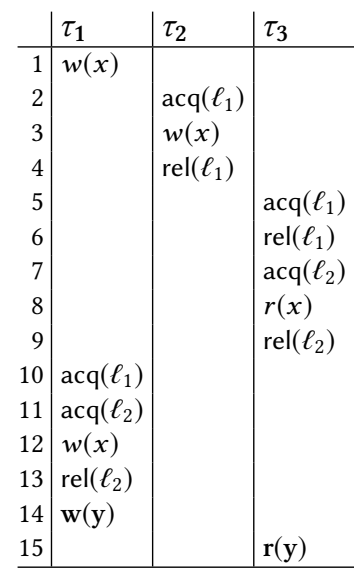

(c) The witness trace.

Fig. 7. (a) The input trace. (b) The partial order $P$ (solid edges) and its closure $Q$ (solid and dashed edges). (c) The witness trace obtained by extending $\operatorname{Max} \operatorname{Min}(Q)$ with the racy pair $\left(e_{6}, e_{16}\right)$.

\subsection{Examples}

We now illustrate the algorithm RaceDecision on a few examples.

Example of a race (Figure 7). Consider the trace $t$ shown in Figure 7a, and the task is to decide whether $\left(e_{6}, e_{16}\right)$ is a predictable race of $t$. The algorithm constructs the causal cones

$$
\text { RCone }_{t}\left(e_{6}, p_{3}\right)=\left\{e_{i}\right\}_{i=1}^{5} \text { and } \text { RCone }_{t}\left(e_{16}, p_{1}\right)=\left\{e_{i}\right\}_{i=8}^{15}
$$

and the partial order $P$ that respects $t$, shown in Figure $7 \mathrm{~b}$ in solid edges. Afterwards, the algorithm computes the closure $Q$ of $P$ by inserting the dashed edges in Figure 7b. In particular, since for the write event $e_{4}$ we have $e_{9}<_{P} e_{4}$ and $e_{9}$ is observed by the read event $e_{14}$, we have $e_{14}<_{Q} e_{4}$ (i.e., this is an observation-closure edge). After this ordering is inserted, for the lock-acquire event $e_{13}$ we have $e_{13}<_{Q} e_{5}$ and $e_{5}$ is a lock-release event on the same lock, we also have $e_{15}<_{Q} e_{3}$, where $e_{15}=\operatorname{match}_{t}\left(e_{13}\right)$ and $e_{3}=\operatorname{match}_{t}\left(e_{5}\right)$ (i.e., this is a lock-closure edge). Now consider the nondeterministic choice made in Line 6 of RaceDecision such that $i=1$. The algorithm also orders $e_{10}<_{Q} e_{11}$ by performing InsertAndClose $\left(Q, e_{10} \rightarrow e_{11}\right)$. Notice that, after this operation, $Q$ is a closed partial order. In addition, the $w(x)$ event $e_{1}$ is unordered with the conflicting events $e_{9}$ and $e_{10}$, i.e., $Q$ is not an M-trace. However, by taking $X_{1}=X \mid p_{1}$ and $X_{2}=X \backslash X_{2}$, we have that width $\left(Q \mid X_{1}\right)=1$ and $Q \mid X_{2}$ is an M-trace. Hence, by Theorem $3.1 Q$ is linearizable to a correct reordering, constructed as the max-min linearization $t^{*}=\operatorname{Max} \operatorname{Min}(Q)$. This illustrates the advantage of our technique over existing methods, as here a correct reordering is exposed even though the initial partial order $P$ contains several pairs of conflicting events that are unordered. Indeed, this race is missed by all HB, WCP, DC and SHB. Finally, the witness trace in is constructed by extending $t^{*}$ with the racy events $e_{6}, e_{16}$, shown in Figure $7 \mathrm{c}$.

Example of a non-race (Figure 8). Consider the trace $t$ shown in Figure 8a, and the task is to decide whether $\left(e_{5}, e_{13}\right)$ is a predictable race of $t$. The algorithm constructs the causal cones

$$
\text { RCone }_{t}\left(e_{5}, p_{3}\right)=\left\{e_{i}\right\}_{i=1}^{4} \text { and } \text { RCone }_{t}\left(e_{13}, p_{1}\right)=\left\{e_{1}\right\} \cup\left\{e_{i}\right\}_{i=7}^{12}
$$




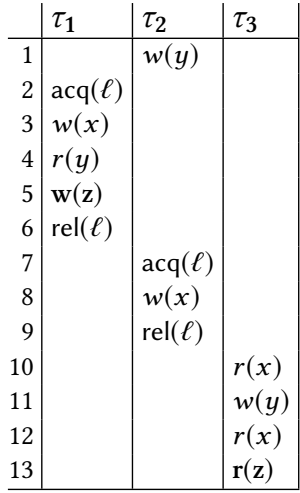

(a) Is $\left(e_{5}, e_{13}\right)$ a race?

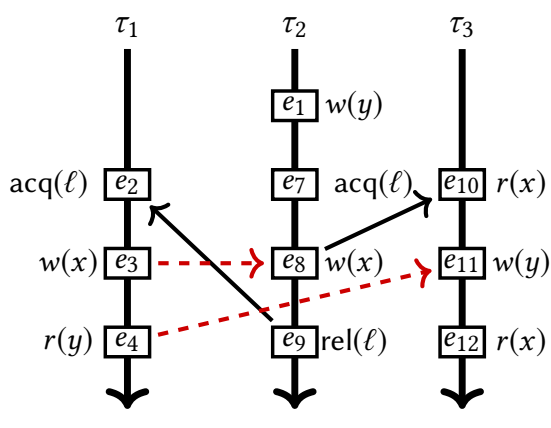

(b) The partial order $P$ is not feasible.

Fig. 8. (a) The input trace. (b) The partial order $P$ (solid edges) and its closure $Q$ (solid and dashed edges).

and the partial order $P$ that respects $t$, shown in Figure 8b in solid edges. Afterwards, the algorithm computes the closure $Q$ of $P$ by inserting the dashed edges in Figure 8b. Observe that $Q$ contains a cycle and thus $P$ is not feasible, hence the algorithm reports that $\left(e_{4}, e_{13}\right)$ is not a race ${ }^{1}$.

Examples from Section 1. Finally, we outline RaceDecision on the two races from Figure 1.

Example from Figure 1a. The algorithm constructs the causal cones

$$
\text { RCone }_{t}\left(e_{2}, p_{2}\right)=\left\{e_{1}\right\} \text { and } \text { RCone }_{t}\left(e_{7}, p_{1}\right)=\left\{e_{i}\right\}_{i=4}^{6}
$$

and the partial order $P$ that respects $t$ by forcing the ordering $e_{6}<_{P} e_{1}$. Note that $P$ is already closed, hence $Q=P$ by the algorithm Closure. Finally, the witness trace is constructed by obtaining the max-min linearization $t^{*}=\operatorname{Max} \operatorname{Min}(Q)$ and extending $t^{*}$ with the racy events $e_{2}, e_{7}$, thereby witnessing the race by the trace $t^{*}=e_{4}, e_{5}, e_{6}, e_{1}, e_{2}, e_{7}$.

Example from Figure $1 b$. The algorithm constructs the causal cones

$$
\text { RCone }_{t}\left(e_{2}, p_{3}\right)=\left\{e_{1}\right\} \text { and } \text { RCone }_{t}\left(e_{14}, p_{1}\right)=\left\{e_{i}\right\}_{i=5}^{13}
$$

and the partial order $P$ that respects $t$ by forcing the orderings $e_{10}<_{P} e_{1}$ and $e_{7}<_{P} e_{12}$. Afterwards, the algorithm computes the closure of $Q$ by inserting $e_{8}<_{Q} e_{11}$. Finally, the witness trace is constructed by obtaining the max-min linearization $t^{*}=\operatorname{Max} \operatorname{Min}(Q)$ and extending $t^{*}$ with the racy events $e_{2}, e_{14}$, thereby witnessing the race by the trace $t^{*}=e_{5}, e_{6}, e_{7}, e_{8}, e_{9}, e_{10}, e_{1}, e_{11}, e_{12}, e_{13}, e_{2}, e_{14}$.

\section{THE FUNCTION PROBLEM IN PRACTICE}

Corollary 4.2 solves the function problem by solving the decision problem on every pair of events of the input trace. Here we present an explicit algorithm for the function problem, called M2, which is the main contribution of this work. Although $\mathrm{M} 2$ does not improve the worst-case complexity, it is faster in practice. The algorithm relies on the following simple lemma.

Lemma 5.1. Consider two conflicting events $e_{1}, e_{2}$ and let $X=$ RCone $_{t}\left(e_{1}, \mathrm{p}\left(e_{2}\right)\right) \cup \mathrm{RCone}_{t}\left(e_{2}, \mathrm{p}\left(e_{1}\right)\right)$. If $X \cap\left\{e_{1}, e_{2}\right\}=\emptyset$ and OpenAcqs $_{t}(X)=\emptyset$ then $\left(e_{1}, e_{2}\right)$ is a predictable race of $t$.

\footnotetext{
${ }^{1}$ While computing the closure different cycles might appear, depending on the order in which the closure rules are applied.
} 
Intuitively, if the conditions of Lemma 5.1 are met, we can postpone the execution of $e_{1}$ in $t$ until $e_{2}$, and the trace witnessing the race is simply $t \mid X \circ e_{1}, e_{2}$.

The algorithm $M 2_{e_{1}}^{p}$. We are now ready to describe an algorithm for partially solving the function problem on an input trace $t$. In particular, we present the algorithm $M 2_{e_{1}}^{p}$ given an event $e_{1} \in \mathcal{E}(t)$ and a process $p \neq p\left(e_{1}\right)$. The algorithm returns the set $\mathcal{Z} \subseteq\left\{e_{1}\right\} \times \mathcal{E}(t) \mid p$ of races detected between $e_{1}$ and events of process $p$. The algorithm simply iterates over all events $e_{2}$ of $p$ in increasing order and computes the causal cone RCone ${ }_{t}\left(e_{2}, \mathrm{p}\left(e_{1}\right)\right)$. Let $X=\mathrm{RCone}_{t}\left(e_{1}, \mathrm{p}\left(e_{2}\right)\right) \cup \mathrm{RCone}_{t}\left(e_{2}, \mathrm{p}\left(e_{1}\right)\right)$. If there are open lock-acquire events in $X$, the algorithm invokes RaceDecision for solving the decision problem on $\left(e_{1}, e_{2}\right)$. Otherwise, a race $\left(e_{1}, e_{2}\right)$ is directly inferred, due to Lemma 5.1. Algorithm 6 gives the formal description. The efficiency of $M 2_{e_{1}}^{p}$ lies on two observations:

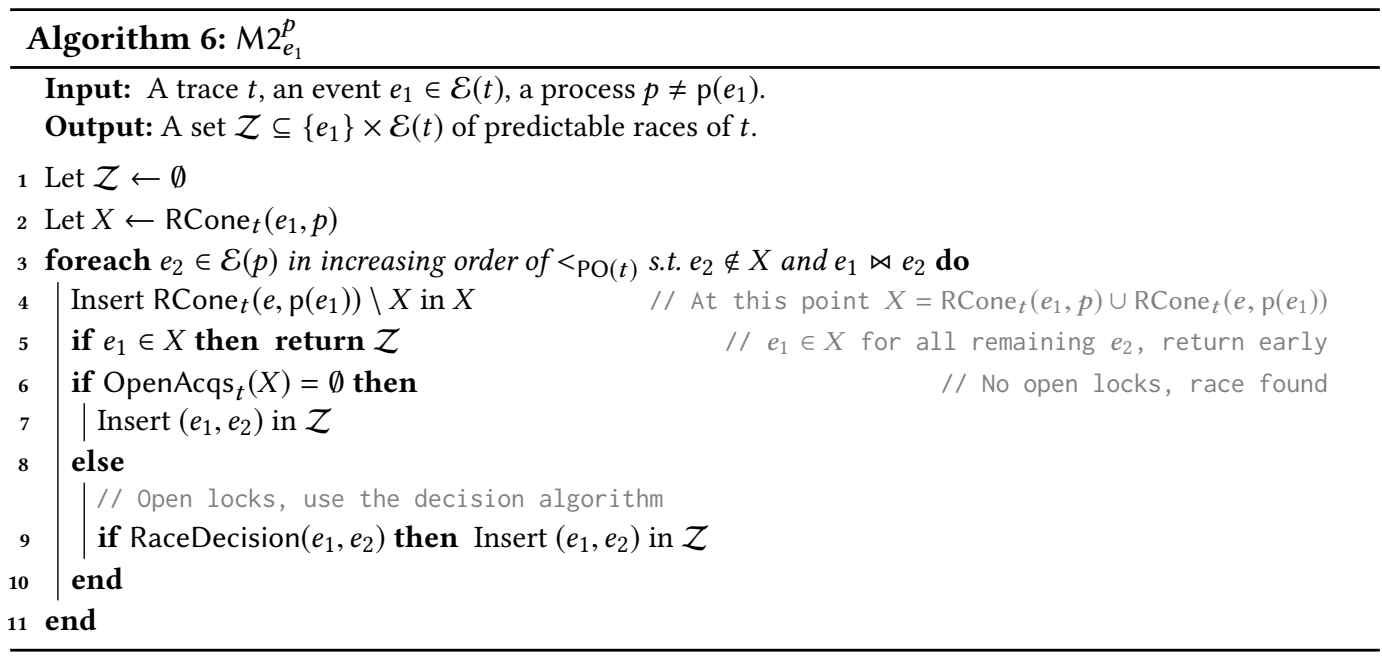

(1) Since critical sections tend to be small, we expect the condition in Line 6 to be False only a few times, thus Lemma 5.1 allows to soundly report a race without constructing a partial order.

(2) The causal cones are closed wrt the program order. That is, given two events $e_{2}$ and $e_{2}^{\prime}$ with $e_{2}<\mathrm{pO} e_{2}^{\prime}$, we have that RCone $e_{t}\left(e_{2}, \mathrm{p}\left(e_{1}\right)\right) \subset \mathrm{RCone}_{t}\left(e_{2}^{\prime}, \mathrm{p}\left(e_{1}\right)\right)$, and thus we only need to consider the difference RCone ${ }_{t}\left(e_{2}^{\prime}, \mathrm{p}\left(e_{1}\right)\right) \backslash$ RCone $_{t}\left(e_{2}, \mathrm{p}\left(e_{1}\right)\right)$ in Line 4 . This decreases the total time for constructing all causal cones from quadratic to linear.

The algorithm M2. Finally, we outline the algorithm $M 2$ for solving the function problem. Given an input trace $t$, the algorithm simply invokes $M 2_{e_{1}}^{p}$ for every event $e_{1} \in \mathcal{E}(t)$ and process $p \neq \mathrm{p}\left(e_{1}\right)$ and obtains the returned race set $\mathcal{Z}_{e_{1}}^{p}$. Since there are $O(n)$ such events, the algorithm makes $O(n)$ invocations. The reported set of predictable races of $t$ is then $\mathcal{Z}=\bigcup_{e_{1}, p} \mathcal{Z}_{e_{1}}^{p}$.

Detecting completeness dynamically. Assume that we execute RaceDecision on input events $e_{1}, e_{2}$ and the algorithm returns False. It can be easily shown that if the following conditions hold, then $\left(e_{1}, e_{2}\right)$ is not a predictable race of $t$ (and hence correctly rejected by RaceDecision).

(1) When computing the relative causal past cones in Line 1 of RaceDecision, no event is added to the cones due to Item 4 of the definition of relative causal past cones.

(2) RaceDecision returns False before executing Line 8. 
Let $C$ be the set of races that are rejected by $M 2$ on input trace $t$ such that at least one of the conditions above does not hold. It follows that $C$ over-approximates the set of false negatives of M2. The algorithm is dynamically complete for $t$ if $C_{t}=\emptyset$.

\section{EXPERIMENTS}

In this section we report on an implementation and experimental evaluation of our techniques.

\subsection{Implementation}

We have implemented our algorithm M2 in Java and evaluated its performance on a standard set of benchmarks. We first discuss some details of the implementation.

Handling dynamic processes creation. In the theoretical part of this paper we have neglected dynamic process creation events. In practice such events are common, and all our benchmark traces contain fork $(i)$ and join $(j)$ events (for forking process $p_{i}$ and joining with process $p_{j}$, respectively) To handle such events, we include in the program order PO the following order relationships: If $e_{1}$ is a fork $(i)$ event and $e_{2}$ is a join $(j)$ event, then we include the order relationship $\left(e_{1}<\mathrm{PO} e_{1}^{\prime}\right)$ and $\left(e_{2}^{\prime}<\mathrm{PO} e_{2}\right)$, where $e_{1}^{\prime}$ is the first event of $p_{i}$ and $e_{2}^{\prime}$ is the last event of $p_{j}$ such that $e_{2}^{\prime}<_{t} e_{2}$.

Optimizations. We make two straightforward optimizations, namely, ignoring non-racy locations and over-approximating the racy events. Recall that $R_{t}(X)$ is the weakest partial order over the events of $t$ that respects $t . R_{t}(X)$ can be constructed efficiently by a single pass of $t$. For the first optimization, we simply remove from $t$ all events to a location $x$ if every pair of conflicting events on $x$ is ordered by $R_{t}(X)$. For the second optimization, we construct the set

$A=\left\{\left(e_{1}, e_{2}\right): e_{1} \bowtie e_{2} \quad\right.$ and $\quad e_{1} \|_{R_{t}(X)} e_{2} \quad$ and $\quad e_{1}, e_{2}$ are not protected by the same lock $\}$. which over-approximates the races of $t$, and, we only consider the pairs $\left(e_{1}, e_{2}\right) \in A$ for races.

\subsection{Experimental Setup}

Benchmarks. Our benchmark set is a standard one found in recent works on race detection [Huang et al. 2014; Kini et al. 2017; Mathur et al. 2018; Yu et al. 2018], and parts of it also exist in other works [Bond et al. 2010; Flanagan and Freund 2009; Roemer et al. 2018; Zhai et al. 2012]. It contains concurrent traces of various sizes, which are concrete executions of concurrent programs taken from standard benchmark suits: (i) the IBM Contest benchmark suite [Farchi et al. 2003], (ii) the Java Grande forum benchmark suite [Smith et al. 2001], (iii) the DaCapo benchmark suite [Blackburn et al. 2006], and (iv) some standalone, real-world software. We have also included the benchmark cryptorsa from the SPEC JVM08 benchmark suite [SPEC 2008] which we have found to be racy. We refer to Table 1 for various interesting statistics on each benchmark trace. The columns $k$ and $n$ denote the number of processes and number of events in each input trace. In each case, $k$ is also used as the bound of the width of the partial orders constructed by our algorithm.

Comparison with $\mathrm{HB}, \mathrm{WCP}, \mathrm{DC}$ and $\mathrm{SHB}$. We compare $\mathrm{M} 2$ against the standard $\mathrm{HB}$, as well as WCP [Kini et al. 2017], DC [Roemer et al. 2018], and SHB [Mathur et al. 2018] which, to our knowledge, are the most recent advances in race prediction. These are partial-order methods based on vector clocks. All implementations are in Java: we rely on the tool Rapid [Mathur et al. 2018] for running $\mathrm{HB}, \mathrm{WCP}$ and $\mathrm{SHB}$, and on our own implementation of DC. To obtain all race reports for an input trace $t$, we use the following process. We construct the corresponding partial order incrementally, by inserting new events in the order they appear in $t$. In addition, we use an extra vector clock $R_{x}, W_{x}$, for every location $x$, which records the vector clock of the last read and write event, respectively, that accessed the respective location. These vector clocks are used to determine 
Table 1. Statistics on our benchmark set.

\begin{tabular}{|c||c|c|c|c|||c||c|c|c|c|}
\hline Benchmark & $n$ & $k$ & \# variables & \# locks & Benchmark & $n$ & $k$ & \# variables & \# locks \\
\hline \hline array & 44 & 2 & 30 & 2 & moldyn & $164 \mathrm{~K}$ & 2 & $1.0 \mathrm{~K}$ & 2 \\
\hline critical & 49 & 3 & 30 & 0 & derby & $1.0 \mathrm{M}$ & 3 & $185 \mathrm{~K}$ & $1.0 \mathrm{~K}$ \\
\hline airtickets & 116 & 2 & 46 & 0 & jigsaw & $3.0 \mathrm{M}$ & 13 & $103 \mathrm{~K}$ & 280 \\
\hline account & 125 & 3 & 41 & 3 & bufwriter & $11 \mathrm{M}$ & 5 & 56 & 1 \\
\hline pingpong & 126 & 4 & 54 & 0 & hsqldb & $18 \mathrm{M}$ & 43 & $946 \mathrm{~K}$ & 412 \\
\hline bbuffer & 322 & 2 & 73 & 2 & cryptorsa & $57 \mathrm{M}$ & 7 & $1.0 \mathrm{M}$ & $8.0 \mathrm{~K}$ \\
\hline mergesort & $3.0 \mathrm{~K}$ & 4 & 621 & 3 & eclipse & $86 \mathrm{M}$ & 14 & $10 \mathrm{M}$ & $8.0 \mathrm{~K}$ \\
\hline bubblesort & $4.0 \mathrm{~K}$ & 10 & 196 & 3 & xalan & $122 \mathrm{M}$ & 6 & $4.0 \mathrm{M}$ & $2.0 \mathrm{~K}$ \\
\hline raytracer & $16 \mathrm{~K}$ & 2 & $3.0 \mathrm{~K}$ & 8 & lusearch & $216 \mathrm{M}$ & 7 & $5.0 \mathrm{M}$ & 118 \\
\hline ftpserver & $48 \mathrm{~K}$ & 10 & $5.0 \mathrm{~K}$ & 304 & - & - & - & - & - \\
\hline
\end{tabular}

whether the current event is racy. After inserting an event $e_{1}$ in the partial order, we iterate over each conflicting event $e_{2}$ that precedes $e_{1}$ in $t$, and determine whether $e_{2}<e_{1}$. If not, we report a race $\left(e_{1}, e_{2}\right)$, and join the vector clock of $e_{1}$ with the vector clock of the corresponding location.

As HB and WCP are only sound on the first race, and DC is unsound, the above process creates, in general, false positives. In order to have a basis for comparison on sound reports, $\left(e_{1}, e_{2}\right)$ is regarded as a reported race by each of these methods only if $M 2$ reports it either as a race, or a possibly false negative (i.e., either $\left(e_{1}, e_{2}\right) \in \mathcal{Z}$ or $\left(e_{1}, e_{2}\right) \in C$, the sets $\mathcal{Z}$ and $C$ as defined in Section 5$)^{2}$.

Race reports. Recall that a race is defined as a pair of events $\left(e_{1}, e_{2}\right)$ of the input trace. However, the interest of the programmer is on the actual code lines $\left(l_{1}, l_{2}\right)$ that these events correspond to. Since long traces typically come from code that executes repeatedly in a loop, we expect to have many racy pairs of events $\left\{\left(e_{1}^{i}, e_{2}^{i}\right)\right\}_{i}$ that correspond to the same racy pair of code lines $\left(l_{1}, l_{2}\right)$, and hence all such pairs $\left(e_{1}^{i}, e_{2}^{i}\right)$ require a single fix. Hence, although the input trace might contain many different event pairs that correspond to the same line pair, these will result in a single race report.

\subsection{Experimental Results}

Our evaluation is summarized in Table 2. The columns Races and Time show the number of reported races, and the time taken, respectively by each method. The column FN reports the size of the set $C$, which gives an upper-bound on the number of false negatives of M2 (see Section 5).

Race detection capability. We see that $\mathrm{M} 2$ is very effective: overall, it discovers hundreds of real races on all benchmarks, regardless of their size and number of processes. In addition, M2 is found complete on all benchmarks (i.e., our over-approximation of the false negatives in column FN always reports at most 0 false negatives). Hence, $M 2$ manages to detect all races in our benchmark set. To our knowledge, this is the first sound technique that reaches such a level of completeness.

On the other hand, the capability of HB, WCP, DC and SHB is more limited, as they all miss several races on several benchmarks. We observe that $\mathrm{WCP}$ catches more races than $\mathrm{HB}$, and DC more races than WCP. This is predicted by theory, as HB races are WCP races [Kini et al. 2017], and WCP races are DC races [Roemer et al. 2018]. On the other hand, although SHB captures provably more races than $\mathrm{HB}, \mathrm{SHB}$ is incomparable with DC. In either case, $\mathrm{M} 2$ captures more races than DC on 10 benchmarks, and than SHB on 6 benchmarks. In addition, on 5 benchmarks (shown in

${ }^{2} \mathrm{SHB}$ is sound on all race reports, and hence this filtering is not performed. 
Table 2. Experimental comparison between HB, WCP, DC, SHB and our algorithm M2. The column FN shows an upper bound on the number of races missed by $\mathrm{M} 2$.

\begin{tabular}{|c||c|c||c|c||c|c||c|c||c|c|c|}
\hline \multicolumn{1}{|l|}{ Benchmark } & \multicolumn{3}{c|}{ HB } & \multicolumn{2}{c|}{ WCP } & \multicolumn{3}{c|}{ DC } & \multicolumn{3}{c|}{ MH } \\
\hline & Races & Time & Races & Time & Races & Time & Races & Time & Races & FN & Time \\
\hline \hline array & 0 & $0.30 \mathrm{~s}$ & 0 & $0.28 \mathrm{~s}$ & 0 & $2.12 \mathrm{~s}$ & 0 & $0.29 \mathrm{~s}$ & 0 & 0 & $0.12 \mathrm{~s}$ \\
\hline critical & 3 & $0.29 \mathrm{~s}$ & 3 & $0.30 \mathrm{~s}$ & 3 & $2.11 \mathrm{~s}$ & 8 & $0.28 \mathrm{~s}$ & 8 & 0 & $0.10 \mathrm{~s}$ \\
\hline airtickets & 3 & $0.32 \mathrm{~s}$ & 3 & $0.31 \mathrm{~s}$ & 3 & $2.10 \mathrm{~s}$ & 4 & $0.31 \mathrm{~s}$ & 4 & 0 & $0.12 \mathrm{~s}$ \\
\hline account & 1 & $0.31 \mathrm{~s}$ & 1 & $0.32 \mathrm{~s}$ & 1 & $2.12 \mathrm{~s}$ & 1 & $0.30 \mathrm{~s}$ & 1 & 0 & $0.12 \mathrm{~s}$ \\
\hline pingpong & 2 & $0.30 \mathrm{~s}$ & 2 & $0.31 \mathrm{~s}$ & 2 & $2.04 \mathrm{~s}$ & 2 & $0.31 \mathrm{~s}$ & 2 & 0 & $0.09 \mathrm{~s}$ \\
\hline bbuffer & 2 & $0.30 \mathrm{~s}$ & 2 & $0.31 \mathrm{~s}$ & 2 & $2.12 \mathrm{~s}$ & 2 & $0.31 \mathrm{~s}$ & 2 & 0 & $0.09 \mathrm{~s}$ \\
\hline mergesort & 1 & $0.36 \mathrm{~s}$ & 1 & $0.41 \mathrm{~s}$ & 1 & $2.16 \mathrm{~s}$ & 1 & $0.37 \mathrm{~s}$ & $\mathbf{2}$ & 0 & $0.18 \mathrm{~s}$ \\
\hline bubblesort & 4 & $0.46 \mathrm{~s}$ & 4 & $0.54 \mathrm{~s}$ & 5 & $2.28 \mathrm{~s}$ & 6 & $0.62 \mathrm{~s}$ & 6 & 0 & $0.71 \mathrm{~s}$ \\
\hline raytracer & 3 & $0.51 \mathrm{~s}$ & 3 & $0.56 \mathrm{~s}$ & 3 & $2.57 \mathrm{~s}$ & 3 & $0.51 \mathrm{~s}$ & 3 & 0 & $0.23 \mathrm{~s}$ \\
\hline ftpserver & 23 & $0.79 \mathrm{~s}$ & 23 & $1.28 \mathrm{~s}$ & 24 & $2.75 \mathrm{~s}$ & 23 & $0.73 \mathrm{~s}$ & $\mathbf{2 6}$ & 0 & $0.88 \mathrm{~s}$ \\
\hline moldyn & 2 & $1.50 \mathrm{~s}$ & 2 & $1.81 \mathrm{~s}$ & 2 & $3.88 \mathrm{~s}$ & 2 & $1.52 \mathrm{~s}$ & 2 & 0 & $1.08 \mathrm{~s}$ \\
\hline derby & 12 & $8.53 \mathrm{~s}$ & 12 & $14.54 \mathrm{~s}$ & 12 & $15.29 \mathrm{~s}$ & 12 & $8.32 \mathrm{~s}$ & 12 & 0 & $7.84 \mathrm{~s}$ \\
\hline jigsaw & 8 & $17.51 \mathrm{~s}$ & 10 & $21.80 \mathrm{~s}$ & 10 & $40.89 \mathrm{~s}$ & 9 & $17.93 \mathrm{~s}$ & $\mathbf{1 1}$ & 0 & $14.65 \mathrm{~s}$ \\
\hline bufwriter & 2 & $48.64 \mathrm{~s}$ & 2 & $2 \mathrm{~m} 0 \mathrm{~s}$ & 2 & $2 \mathrm{~m} 59 \mathrm{~s}$ & 2 & $47.71 \mathrm{~s}$ & 2 & 0 & $57.37 \mathrm{~s}$ \\
\hline hsqldb & 4 & $3 \mathrm{~m} 53 \mathrm{~s}$ & 4 & $3 \mathrm{~m} 5 \mathrm{~s}$ & 5 & $4 \mathrm{~m} 23 \mathrm{~s}$ & 9 & $3 \mathrm{~m} 53 \mathrm{~s}$ & 9 & 0 & $7 \mathrm{~m} 1 \mathrm{~s}$ \\
\hline cryptorsa & 5 & $3 \mathrm{~m} 42 \mathrm{~s}$ & 5 & $3 \mathrm{~m} 0 \mathrm{~s}$ & 7 & $6 \mathrm{~m} 58 \mathrm{~s}$ & 5 & $3 \mathrm{~m} 29 \mathrm{~s}$ & 7 & 0 & $6 \mathrm{~m} 6 \mathrm{~s}$ \\
\hline eclipse & 33 & $8 \mathrm{~m} 1 \mathrm{~s}$ & 34 & $7 \mathrm{~m} 0 \mathrm{~s}$ & 39 & $14 \mathrm{~m} 44 \mathrm{~s}$ & 54 & $7 \mathrm{~m} 11 \mathrm{~s}$ & $\mathbf{6 7}$ & 0 & $45 \mathrm{~m} 23 \mathrm{~s}$ \\
\hline xalan & 7 & $8 \mathrm{~m} 58 \mathrm{~s}$ & 7 & $8 \mathrm{~m} 25 \mathrm{~s}$ & 9 & $20 \mathrm{~m} 12 \mathrm{~s}$ & 11 & $9 \mathrm{~m} 8 \mathrm{~s}$ & $\mathbf{1 5}$ & 0 & $7 \mathrm{~m} 15 \mathrm{~s}$ \\
\hline lusearch & 30 & $16 \mathrm{~m} 4 \mathrm{~s}$ & 30 & $9 \mathrm{~m} 59 \mathrm{~s}$ & 30 & $2 \mathrm{~h} 49 \mathrm{~m} 6 \mathrm{~s}$ & 52 & $15 \mathrm{~m} 28 \mathrm{~s}$ & 52 & 0 & $8 \mathrm{~m} 9 \mathrm{~s}$ \\
\hline \hline Total & $\mathbf{1 4 5}$ & $\mathbf{4 2 m 0 s}$ & $\mathbf{1 4 8}$ & $\mathbf{3 4 m 1 4 s}$ & $\mathbf{1 6 0}$ & $\mathbf{3 h 3 9 m}$ & $\mathbf{2 0 6}$ & $\mathbf{4 0 m 3 1 s}$ & $\mathbf{2 3 1}$ & $\mathbf{0}$ & $\mathbf{1 h 1 5 m}$ \\
\hline
\end{tabular}

Table 3. Mean and maximum distances (in number of intervening events) on races detected by M2.

\begin{tabular}{|c||c|c|}
\hline Benchmark & Mean Distance & Max Distance \\
\hline \hline ftpserver & 939 & $12 \mathrm{~K}$ \\
\hline jigsaw & $1 \mathrm{~K}$ & $4 \mathrm{~K}$ \\
\hline hsqldb & $92 \mathrm{~K}$ & $1 \mathrm{M}$ \\
\hline cryptorsa & $1 \mathrm{M}$ & $8 \mathrm{M}$ \\
\hline eclipse & $11 \mathrm{M}$ & $53 \mathrm{M}$ \\
\hline xalan & $2.0 \mathrm{~K}$ & $43 \mathrm{~K}$ \\
\hline lusearch & $44 \mathrm{M}$ & $125 \mathrm{M}$ \\
\hline
\end{tabular}

Table 4. Racy locations missed by each method. For HB, WCP, DC, SHB the numbers are lowerbounds. For M2, the numbers are upper-bounds.

\begin{tabular}{|c||c|c|c|c|c|}
\hline Benchmark & HB & WCP & DC & SHB & M2 \\
\hline \hline ftpserver & 3 & 3 & 3 & 3 & 0 \\
\hline jigsaw & 2 & 0 & 0 & 2 & 0 \\
\hline cryptorsa & 1 & 1 & 0 & 1 & 0 \\
\hline eclipse & 16 & 11 & 8 & 10 & 0 \\
\hline xalan & 3 & 2 & 2 & 2 & 0 \\
\hline lusearch & 11 & 11 & 11 & 0 & 0 \\
\hline \hline Total & $\mathbf{3 6}$ & $\mathbf{2 8}$ & $\mathbf{2 4}$ & $\mathbf{1 8}$ & $\mathbf{0}$ \\
\hline
\end{tabular}

bold), M2 captures more races than any other algorithm. In total, M2 detects 71 more races than DC and 25 more races than SHB.

We also remark that our algorithm provides more information than the baseline methods even on benchmarks where the number of reported races is the same. This is because M2 manages to detect that the reports in such benchmarks are complete (i.e., no races are missed).

In terms of race distances, we have found that $\mathrm{M} 2$ is able to detect races that are very far apart in the input trace. We refer to Table 3 for a few interesting examples, where the distance of a race $\left(e_{1}, e_{2}\right)$ is counted as the number of intervening events between $e_{1}$ and $e_{2}$ in the input trace. For instance, 
in lusearch, the maximum race distance detected by $\mathrm{M} 2$ is $125 \mathrm{M}$ events. Note that, in general, the same memory location can be reported as racy by many data-race pairs $\left(e_{1}, e_{2}\right)$. To assess the significance of the new races detected by $\mathrm{M} 2$, we have also computed the number of racy memory locations that are missed by each method. We see that $\mathrm{M} 2$ misses 0 memory locations (i.e., it detects all racy memory locations). For each of HB, WCP, DC and SHB, this number has been computed by counting how many locations have been detected by $\mathrm{M} 2$ and missed by the corresponding method. We refer to Table 4 for the cases where at least one method missed some racy memory location. In total, each of the baseline methods misses tens of racy memory locations. Finally, we have also computed location-specific race distances, as follows. For each location $x$, we computed the minimum distance $d_{x}$ between all races on location $x$. Hence, $d_{x}$ holds the smallest distance of a race which reveals that the location $x$ is racy. The mean and maximum location-specific race distances in eclipse are $3 \mathrm{M}$ and $38 \mathrm{M}$ events, respectively, while in lusearch, they are $33 \mathrm{M}$ and $125 \mathrm{M}$ events, respectively. These numbers indicate that windowing techniques, which are typically restricted to windows of a few hundreds/thousands of events, are likely to produce highly incomplete results, and even fail to detect that certain memory locations are racy. Similar observations have also been made in recent works [Kini et al. 2017; Roemer et al. 2018].

Scalability. We see that $\mathrm{M} 2$ has comparable running time to the baseline methods, and is sometimes faster. One clear exception is on eclipse, where $M 2$ requires about $45 \mathrm{~m}$, whereas the other methods spend between $7 \mathrm{~m}$ and $14 \mathrm{~m}$. However, this is the benchmark on which all other methods miss both the most races (at least 13, see Table 2) and the most racy memory locations (at least 8, see Table 4) Hence, the completeness of our race reports comes at a relatively small increase in running time.

To better understand the efficiency of $M 2$, recall that its worst-case complexity is a product of two factors, $O(\alpha \cdot \beta)$, where $\alpha$ is the number of calls to RaceDecision for verifying race pairs, and $\beta$ is the time taken by Closure to compute the closure of the underlying partial order $P$. In the worst case, both $\alpha$ and $\beta$ are $\Theta\left(n^{2}\right)$ (ignoring log-factors). In practice, we have observed that M2 resorts on calling RaceDecision only a small number of times, hence $\alpha$ is small. This illustrates the practical advantage of M2 over the naive approach that just uses RaceDecision on all $\left(\begin{array}{l}n \\ 2\end{array}\right)$ event pairs. In addition, we can express $\beta$ as roughly $\beta=n+m \cdot \gamma$, where $m$ is the number of edges inserted in $P$ during closure, and $\gamma$ is the time spent for each such edge. Using our data structure DS for representing $P$, we have $\gamma=O(\log n)$, and, although $m=\Theta\left(n^{2}\right)$ in the worst-case, we have observed that $m$ behaves as a constant in practice.

Finally, we note that the baseline methods may admit further engineering optimizations that reduce their running time. Such optimizations exist for $\mathrm{HB}$, but we are unaware of any attempts to optimize WCP, DC or SHB further. In any case, although our tool is not faster, the take-home message is well-supported: M2 makes sound and effectively complete race predictions, at running times comparable to the theoretically fastest, yet highly incomplete, state-of-the-art methods.

\section{RELATED WORK}

In this section we briefly review related work on dynamic race detection.

Predictive analyses. Predictive techniques aim at inferring program behavior simply by looking at given traces. In the context of race detection, the CP partial order [Smaragdakis et al. 2012] and WCP partial order [Kini et al. 2017] are sound but incomplete predictive techniques based on partial orders. A somewhat different approach was proposed recently in [Roemer et al. 2018], based on the DC partial order. DC imposes fewer orderings than WCP, but is generally unsound. To create sound warnings, a DC-race is followed by a vindication phase, which is sound but incomplete. 
Other works in this domain include [Huang et al. 2014; Liu et al. 2016; Said et al. 2011; Wang et al. 2009], which typically approach the problem based on SAT/SMT encodings. These works are sound and complete in theory, but require exponential time. In practice, techniques such as windowing make these methods operate fast, at the cost of sacrificing completeness. Predictive techniques have also been used for atomicity violations and synchronization errors [Chen et al. 2008; Huang and Rauchwerger 2015; Sen et al. 2005; Sorrentino et al. 2010], as well as in lock-based communication [Farzan et al. 2009; Kahlon et al. 2005; Sorrentino et al. 2010].

Happens-before techniques. A large pool of race detectors are based on Lamport's happensbefore relation [Lamport 1978], which yields the HB partial order. HB can be computed in linear time [Mattern 1989] and has been the technical basis behind many approaches [Bond et al. 2010; Christiaens and Bosschere 2001; Flanagan and Freund 2009; Pozniansky and Schuster 2003; Schonberg 1989]. The tradeoff between runtime and space usage in race-detection using the happensbefore relation was studied in [Banerjee et al. 2006]. Recently, the SHB partial order was proposed as an extension to HB in order to effectively detect multiple races per trace [Mathur et al. 2018].

Lockset-based techniques. A lockset of a variable is the set of locks that guard critical regions in which the variable is accessed. Lockset-based techniques report races by comparing the locksets of the variables accessed by the corresponding events. They were introduced in [Dinning and Schonberg 1991] and equipped by the tool of [Savage et al. 1997]. Lockset-based techniques tend to produce many false positives and this problem has been targeted by various enhancements such as random testing [Sen 2008] and static analysis [Choi et al. 2002; von Praun and Gross 2001].

Other approaches. To reduce unsound reports, lockset-based techniques have been combined with happens-before techniques [Elmas et al. 2007; O'Callahan and Choi 2003; Yu et al. 2005]. Other approaches include statistical techniques [Bond et al. 2010; Marino et al. 2009] and static race-detectors [Naik et al. 2006; Pratikakis et al. 2011; Voung et al. 2007]. Recently, [Genç et al. 2019] applied a combination of static and dynamic techniques to allow for correct reorderings in which the observation of some read events is allowed to differ between the input and witness trace, as long as the read does not affect the control-flow of the respective thread. Such static information can be directly incorporated in the techniques we have developed in this paper, and is left for interesting follow-up work. Dynamic race detection has also been studied under structured parallelism [Raman et al. 2012] and relaxed memory models [Kim et al. 2009; Lidbury and Donaldson 2017].

\section{CONCLUSION}

We have presented M2: a new polynomial-time algorithm for the problem that has no false positives. In addition, our algorithm is complete for input traces that consist of two processes, i.e., it provably detects all races in the trace. We have also developed criteria for detecting completeness dynamically, even in the case of more than two processes. Our experimental validation found that $\mathrm{M} 2$ is very effective in practice, as it soundly reported all races in the input benchmark set. Although M2 is not theoretically complete in the general case, we believe that its completeness guarantee on two processes provides some explanation of why it performs so well in practice.

\section{A INCREMENTAL DAG REACHABILITY}

In this section we target the problem of solving incremental reachability on Directed Acyclic Graphs (DAGs). Informally, we are given a DAG and an online sequence of (i) edge-insertion and (ii) reachability query operations. The task is to answer each reachability query correctly, accounting for all preceding edge-insertion operations. Here we develop a data structure DS for solving the problem efficiently on DAGs of small width. In the main paper we use DS to compute 
the closure of partial orders efficiently. We expect that DS might be of relevance also to other race-detection techniques that are graph-based.

Directed acyclic graphs of small width. Let $G=(V, E)$ be a DAG and $E^{*}$ be the transitive closure of $E$. Note that $E^{*}$ is a partial order, and we let width $(G)=$ width $\left(E^{*}\right)$. Our focus is on DAGs of small width, i.e., we take width $(G)=k=O(1)$. For $u, v \in V$, we write $u \rightsquigarrow v$ if $v$ is reachable from $u$. We represent $G$ as $k$ (totally ordered) chains with extra edges between them. We let $V \subseteq[k] \times[n]$, so that a node of $G$ is represented as a pair $(i, j)$, meaning that it is the $j$-th node in the $i$-th chain For two nodes $\left\langle i, j_{1}\right\rangle,\left\langle i, j_{2}\right\rangle \in V$ with $j_{2}=j_{1}+1$, we have $\left(\left(i, j_{1}\right),\left(i, j_{2}\right)\right) \in E$. Given two nodes $\left\langle i, j_{1}\right\rangle,\left\langle i, j_{2}\right\rangle$, we say that $\left\langle i, j_{1}\right\rangle$ is higher than $\left\langle i, j_{2}\right\rangle$ if $j_{1} \leq j_{2}$. In such a case, we say that $\left\langle i, j_{2}\right\rangle$ is lower than $\left\langle i, j_{1}\right\rangle$. The edge set is represented as a set of arrays Out $i_{i_{1}} \rightarrow[n] \cup\{\infty\}$, where $i_{1}, i_{2} \in[k]$ and $i_{1} \neq i_{2}$. We have that Out $i_{i_{1}}^{i_{2}}\left[j_{1}\right]=j_{2} \in[k]$ iff $\left(\left\langle i_{1}, j_{1}\right\rangle,\left\langle i_{2}, j_{2}\right\rangle\right) \in E$. Note that since $k=O(1)$, such a representation requires $O(n)$ space even if $G$ is a dense graph.

Incremental reachability on DAGs of small width. The incremental reachability problem on a DAG $G=(V, E)$ is defined on an online sequence of operations of the following types.

(1) An insert $(u, v)$ operation, such that $v \not \leftrightarrow u$, inserts the edge $u, v$ in $G$.

(2) A query $(u, v)$ operation returns True iff $u \rightsquigarrow v$.

(3) A successor $(u, i)$ operation returns the highest successor of $u$ in the $i$-th chain.

(4) A predecessor $(u, i)$ operation returns the lowest predecessor of $u$ in the $i$-th chain.

The task is to answer query operations correctly, taking into consideration all preceding insert operations. Note that the width of $G$ does not increase after any operation. We will present a data structure that handles each such query in $O(\log n)$ time. Our data structure is based on the dynamic suffix minima problem, presented below.

Dynamic suffix minima and Fenwick-trees. The dynamic suffix minima problem is defined given an integer array $A$ of length $n$, and an online sequence of operations of the following types.

(1) An update $(i, x)$ operation, for $1 \leq i \leq n$ and $x \in \mathbb{Z}$, sets $A[i]=x$.

(2) $\mathrm{A} \min (i)$ operation, for $1 \leq i \leq n$, returns $\min _{i \leq j \leq n} A[j]$.

(3) An $\arg \operatorname{leq}(i)$ operation, for $1 \leq i \leq n$, returns $\max _{j: A[j] \leq i} j$.

The task is to answer min and arg leq operations correctly, taking into consideration all preceding update operations. The Fenwick-tree data structure solves the dynamic suffix minima problem in $O(\log n)$ time per operation, after $O(n)$ preprocessing time [Fenwick 1994].

The data structure DS for solving the incremental reachability problem. We are now ready to describe our data structure DS for solving the incremental reachability problem given a DAG $G=(V, E)$ of width $k$, and an online sequence $\Sigma$ of insert and query operations. We consider that $G$ is given in a sparse representation form, where outgoing edges are represented using the arrays Out $_{i_{1}}^{i_{2}}$, for each $i_{1}, i_{2} \in[k]$ and $i_{1} \neq i_{2}$.

In the initialization phase, DS performs the following steps. For each $i_{1}, i_{2} \in[k]$ such that $i_{1} \neq i_{2}$, we initialize a Fenwick-tree data structure FenwickTree $i_{i_{1}}^{i_{2}}$ with array Out $i_{i_{1}}^{i_{2}}$. This data structure stores forward reachability information from nodes of the $i_{1}$-th chain to nodes in the $i_{2}$ chain, by maintaining the invariant that FenwickTree $i_{i_{1}}^{i_{2}} \cdot \min \left(j_{1}\right)=j_{2}$ iff $\left\langle i_{2}, j_{2}\right\rangle$ is the highest node of the $i_{2}$-th chain reachable from $\left\langle i_{1}, j_{2}\right\rangle$. A successor $\left(\left\langle i_{1}, j_{1}\right\rangle, i\right)$ (resp., predecessor $\left(\left\langle i_{1}, j_{1}\right\rangle, i\right)$ ) operation is handled by DS by returning FenwickTree $i_{1} \cdot \min \left(j_{1}\right)\left(\right.$ resp., FenwickTree $\left.i_{i_{1}}^{i} \cdot \arg \operatorname{leq}\left(j_{1}\right)\right)$. Finally, the 
operations DS.insert and DS.query are handled by Algorithm 7 and Algorithm 8, respectively. The following lemma establishes the correctness and complexity of the data structure DS.

Lemma A.1. Let $\Sigma$ be an online sequence of incremental reachability operations. The data structure DS correctly handles $\Sigma$ and spends ( $i) O(n)$ preprocessing time and (ii) $O(\log n)$ time per operation.
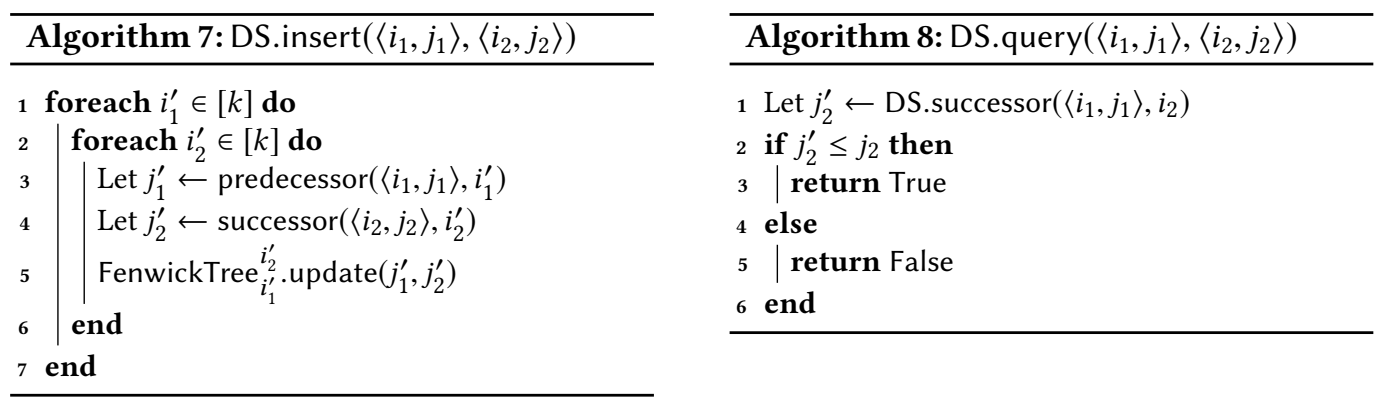

\section{B DEFINITION OF HB, SHB, WCP AND DC}

For the sake of completeness, here we give the definitions of the partial orders HB, SHB, WCP and DC, based on [Kini et al. 2017; Mathur et al. 2018; Roemer et al. 2018]. In each case, we consider given a trace $t$, and the respective partial order is over the set of events $\mathcal{E}(t)$ of $t$.

The HB partial order is the smallest partial order that satisfies the following conditions .

(1) $\mathrm{HB} \sqsubseteq \mathrm{PO}$.

(2) For every lock-acquire and lock-release events acq and rel such that rel $<_{t}$ acq, if acq $\bowtie$ rel then rel $<$ HB acq.

The SHB partial order is the smallest partial order that satisfies the following conditions.

(1) $\mathrm{SHB} \sqsubseteq \mathrm{HB}$.

(2) For every read event $r$ we have $O_{t}(r)<_{\mathrm{SHB}} r$. Recall that $O_{t}(r)$ is the observation of $r$ in $t$.

The WCP partial order is the smallest partial order that satisfies the following conditions.

(1) $\mathrm{WCP} \sqsubseteq \mathrm{PO}$.

(2) For every lock-release event rel and write/read event $e$ such that rel $<_{t} e$, if (i) $e$ is protected by a lock $\ell=\operatorname{loc}\left(\right.$ rel) and (ii) $e$ conflicts with an event in the critical section of rel, then rel $<_{W C P} e$.

(3) For every two lock-release events rel ${ }_{1}, \mathrm{rel}_{2}$ such that $\mathrm{rel}_{1}<_{t} \mathrm{rel}_{2}$, if the critical sections of rel and rel $_{2}$ contain WCP-ordered events, then rel $_{1}<\mathrm{WCP}_{\mathrm{Wel}}$.

(4) WCP is closed under left and right composition with HB.

The DC partial order is the smallest partial order that satisfies conditions 1, 2 and 3 of WCP.

\section{ACKNOWLEDGMENTS}

I am grateful to Umang Mathur for his valuable assistance in the experimental part of the paper, to Viktor Kunčak for his insightful comments in earlier drafts, and to anonymous reviewers for their constructive feedback. This work is partly supported by the Austrian Science Fund (FWF) Schrödinger grant J-4220. 


\section{REFERENCES}

Utpal Banerjee, Brian Bliss, Zhiqiang Ma, and Paul Petersen. 2006. A Theory of Data Race Detection. In Proceedings of the 2006 Workshop on Parallel and Distributed Systems: Testing and Debugging (PADTAD '06). ACM, New York, NY, USA, 69-78. https://doi.org/10.1145/1147403.1147416

Stephen M. Blackburn, Robin Garner, Chris Hoffmann, Asjad M. Khang, Kathryn S. McKinley, Rotem Bentzur, Amer Diwan, Daniel Feinberg, Daniel Frampton, Samuel Z. Guyer, Martin Hirzel, Antony Hosking, Maria Jump, Han Lee, J. Eliot B. Moss, Aashish Phansalkar, Darko Stefanović, Thomas VanDrunen, Daniel von Dincklage, and Ben Wiedermann. 2006. The DaCapo Benchmarks: Java Benchmarking Development and Analysis. In OOPSLA.

Michael D. Bond, Katherine E. Coons, and Kathryn S. McKinley. 2010. PACER: Proportional Detection of Data Races. In Proceedings of the 31st ACM SIGPLAN Conference on Programming Language Design and Implementation (PLDI '10). ACM, New York, NY, USA, 255-268. https://doi.org/10.1145/1806596.1806626

Feng Chen and Grigore Roşu. 2007. Parametric and Sliced Causality. In Proceedings of the 19th International Conference on Computer Aided Verification (CAV'07). Springer-Verlag, Berlin, Heidelberg, 240-253. http://dl.acm.org/citation.cfm?id= 1770351.1770387

Feng Chen, Traian Florin Serbanuta, and Grigore Rosu. 2008. jPredictor: A Predictive Runtime Analysis Tool for Java. In Proceedings of the 30th International Conference on Software Engineering (ICSE '08). ACM, New York, NY, USA, 221-230. https://doi.org/10.1145/1368088.1368119

Jong-Deok Choi, Keunwoo Lee, Alexey Loginov, Robert O’Callahan, Vivek Sarkar, and Manu Sridharan. 2002. Efficient and Precise Datarace Detection for Multithreaded Object-oriented Programs. In Proceedings of the ACM SIGPLAN 2002 Conference on Programming Language Design and Implementation (PLDI '02). ACM, New York, NY, USA, 258-269. https://doi.org/10.1145/512529.512560

Mark Christiaens and Koenraad De Bosschere. 2001. TRaDe: Data Race Detection for Java. In Proceedings of the International Conference on Computational Science-Part II (ICCS '01). Springer-Verlag, London, UK, UK, 761-770. http://dl.acm.org/ citation.cfm?id=645456.654536

Anne Dinning and Edith Schonberg. 1991. Detecting Access Anomalies in Programs with Critical Sections. In Proceedings of the 1991 ACM/ONR Workshop on Parallel and Distributed Debugging (PADD '91). ACM, New York, NY, USA, 85-96. https://doi.org/10.1145/122759.122767

Tayfun Elmas, Shaz Qadeer, and Serdar Tasiran. 2007. Goldilocks: A Race and Transaction-aware Java Runtime. In Proceedings of the 28th ACM SIGPLAN Conference on Programming Language Design and Implementation (PLDI '07). ACM, New York, NY, USA, 245-255. https://doi.org/10.1145/1250734.1250762

Eitan Farchi, Yarden Nir, and Shmuel Ur. 2003. Concurrent Bug Patterns and How to Test Them. In Proceedings of the 17th International Symposium on Parallel and Distributed Processing (IPDPS '03). IEEE Computer Society, Washington, DC, USA, 286.2-. http://dl.acm.org/citation.cfm?id=838237.838485

Azadeh Farzan, P. Madhusudan, and Francesco Sorrentino. 2009. Meta-analysis for Atomicity Violations Under Nested Locking. In Proceedings of the 21st International Conference on Computer Aided Verification (CAV'09). Springer-Verlag, Berlin, Heidelberg, 248-262. https://doi.org/10.1007/978-3-642-02658-4_21

Peter M. Fenwick. 1994. A new data structure for cumulative frequency tables. Software: Practice and Experience 24, 3 (1994), 327-336. https://doi.org/10.1002/spe.4380240306

Cormac Flanagan and Stephen N. Freund. 2009. FastTrack: Efficient and Precise Dynamic Race Detection. In Proceedings of the 30th ACM SIGPLAN Conference on Programming Language Design and Implementation (PLDI'09). ACM, New York, NY, USA, 121-133. https://doi.org/10.1145/1542476.1542490

Kaan Genç, Jake Roemer, Yufan Xu, and Michael D. Bond. 2019. Dependence-Aware, Unbounded Sound Predictive Race Detection (OOPSLA 2019). To appear.

Jim Gray. 1985. Why Do Computers Stop And What Can Be Done About It? Büroautomation (1985), 128-145.

D. P. Helmbold, C. E. McDowell, and Jian-Zhong Wang. 1991. Detecting data races from sequential traces. In Proceedings of the Twenty-Fourth Annual Hawaii International Conference on System Sciences, Vol. ii. 408-417 vol.2. https://doi.org/10. 1109/HICSS.1991.184003

Jeff Huang, Patrick O’Neil Meredith, and Grigore Rosu. 2014. Maximal Sound Predictive Race Detection with Control Flow Abstraction. In Proceedings of the 35th ACM SIGPLAN Conference on Programming Language Design and Implementation (PLDI '14). ACM, New York, NY, USA, 337-348. https://doi.org/10.1145/2594291.2594315

Jeff Huang and Lawrence Rauchwerger. 2015. Finding Schedule-sensitive Branches. In Proceedings of the 2015 10th foint Meeting on Foundations of Software Engineering (ESEC/FSE 2015). ACM, New York, NY, USA, 439-449. https://doi.org/10. $1145 / 2786805.2786840$

Vineet Kahlon, Franjo Ivančić, and Aarti Gupta. 2005. Reasoning About Threads Communicating via Locks. In Proceedings of the 17th International Conference on Computer Aided Verification (CAV'05). Springer-Verlag, Berlin, Heidelberg, 505-518. https://doi.org/10.1007/11513988_49 
KyungHee Kim, Tuba Yavuz-Kahveci, and Beverly A. Sanders. 2009. Precise Data Race Detection in a Relaxed Memory Model Using Heuristic-Based Model Checking. In Proceedings of the 2009 IEEE/ACM International Conference on Automated Software Engineering (ASE '09). IEEE Computer Society, Washington, DC, USA, 495-499. https://doi.org/10.1109/ASE. 2009.82

Dileep Kini, Umang Mathur, and Mahesh Viswanathan. 2017. Dynamic Race Prediction in Linear Time. In Proceedings of the 38th ACM SIGPLAN Conference on Programming Language Design and Implementation (PLDI 2017). ACM, New York, NY, USA, 157-170. https://doi.org/10.1145/3062341.3062374

Leslie Lamport. 1978. Time, Clocks, and the Ordering of Events in a Distributed System. Commun. ACM 21, 7 (July 1978), 558-565. https://doi.org/10.1145/359545.359563

Christopher Lidbury and Alastair F. Donaldson. 2017. Dynamic Race Detection for C++11. In Proceedings of the 44th ACM SIGPLAN Symposium on Principles of Programming Languages (POPL 2017). ACM, New York, NY, USA, 443-457. https://doi.org/10.1145/3009837.3009857

Peng Liu, Omer Tripp, and Xiangyu Zhang. 2016. IPA: Improving Predictive Analysis with Pointer Analysis. In Proceedings of the 25th International Symposium on Software Testing and Analysis (ISSTA 2016). ACM, New York, NY, USA, 59-69. https://doi.org/10.1145/2931037.2931046

Shan Lu, Soyeon Park, Eunsoo Seo, and Yuanyuan Zhou. 2008. Learning from Mistakes: A Comprehensive Study on Real World Concurrency Bug Characteristics. SIGOPS Oper. Syst. Rev. 42, 2 (March 2008), 329-339. https://doi.org/10.1145/ 1353535.1346323

Daniel Marino, Madanlal Musuvathi, and Satish Narayanasamy. 2009. LiteRace: Effective Sampling for Lightweight Data-race Detection. In Proceedings of the 30th ACM SIGPLAN Conference on Programming Language Design and Implementation (PLDI '09). ACM, New York, NY, USA, 134-143. https://doi.org/10.1145/1542476.1542491

Umang Mathur, Dileep Kini, and Mahesh Viswanathan. 2018. What Happens-after the First Race? Enhancing the Predictive Power of Happens-before Based Dynamic Race Detection. Proc. ACM Program. Lang. 2, OOPSLA, Article 145 (Oct. 2018 ), 29 pages. https://doi.org/10.1145/3276515

Friedemann Mattern. 1989. Virtual Time and Global States of Distributed Systems. In Parallel and Distributed Algorithms: proceedings of the International Workshop on Parallel \& Distributed Algorithms, M. Cosnard et. al. (Ed.). Elsevier Science Publishers B. V., 215-226.

A Mazurkiewicz. 1987. Trace Theory. In Advances in Petri Nets 1986, Part II on Petri Nets: Applications and Relationships to Other Models of Concurrency. Springer-Verlag New York, Inc., 279-324.

Madanlal Musuvathi, Shaz Qadeer, Thomas Ball, Gerard Basler, Piramanayagam Arumuga Nainar, and Iulian Neamtiu. 2008. Finding and Reproducing Heisenbugs in Concurrent Programs. In Proceedings of the 8th USENIX Conference on Operating Systems Design and Implementation (OSDI'08). USENIX Association, Berkeley, CA, USA, 267-280. http: //dl.acm.org/citation.cfm?id=1855741.1855760

Mayur Naik, Alex Aiken, and John Whaley. 2006. Effective Static Race Detection for Java. In Proceedings of the 27th ACM SIGPLAN Conference on Programming Language Design and Implementation (PLDI '06). ACM, New York, NY, USA, 308-319. https://doi.org/10.1145/1133981.1134018

Robert O’Callahan and Jong-Deok Choi. 2003. Hybrid Dynamic Data Race Detection. SIGPLAN Not. 38, 10 (June 2003), 167-178. https://doi.org/10.1145/966049.781528

Andreas Pavlogiannis. 2019. Fast, Sound and Effectively Complete Dynamic Race Detection. (2019). arXiv:arXiv:1901.08857

Eli Pozniansky and Assaf Schuster. 2003. Efficient On-the-fly Data Race Detection in Multithreaded C++ Programs. SIGPLAN Not. 38, 10 (June 2003), 179-190. https://doi.org/10.1145/966049.781529

Polyvios Pratikakis, Jeffrey S. Foster, and Michael Hicks. 2011. LOCKSMITH: Practical Static Race Detection for C. ACM Trans. Program. Lang. Syst. 33, 1, Article 3 (Jan. 2011), 55 pages. https://doi.org/10.1145/1889997.1890000

Raghavan Raman, Jisheng Zhao, Vivek Sarkar, Martin Vechev, and Eran Yahav. 2012. Scalable and Precise Dynamic Datarace Detection for Structured Parallelism. In Proceedings of the 33rd ACM SIGPLAN Conference on Programming Language Design and Implementation (PLDI '12). ACM, New York, NY, USA, 531-542. https://doi.org/10.1145/2254064.2254127

Jake Roemer, Kaan Genç, and Michael D. Bond. 2018. High-coverage, Unbounded Sound Predictive Race Detection. In Proceedings of the 39th ACM SIGPLAN Conference on Programming Language Design and Implementation (PLDI 2018). ACM, New York, NY, USA, 374-389. https://doi.org/10.1145/3192366.3192385

Mahmoud Said, Chao Wang, Zijiang Yang, and Karem Sakallah. 2011. Generating Data Race Witnesses by an SMT-based Analysis. In Proceedings of the Third International Conference on NASA Formal Methods (NFM'11). Springer-Verlag, Berlin, Heidelberg, 313-327. http://dl.acm.org/citation.cfm?id=1986308.1986334

Stefan Savage, Michael Burrows, Greg Nelson, Patrick Sobalvarro, and Thomas Anderson. 1997. Eraser: A Dynamic Data Race Detector for Multithreaded Programs. ACM Trans. Comput. Syst. 15, 4 (Nov. 1997), 391-411. https://doi.org/10.1145/ 265924.265927

D. Schonberg. 1989. On-the-fly Detection of Access Anomalies. In Proceedings of the ACM SIGPLAN 1989 Conference on Programming Language Design and Implementation (PLDI '89). ACM, New York, NY, USA, 285-297. https://doi.org/10. 
1145/73141.74844

Koushik Sen. 2008. Race Directed Random Testing of Concurrent Programs. In Proceedings of the 29th ACM SIGPLAN Conference on Programming Language Design and Implementation (PLDI '08). ACM, New York, NY, USA, 11-21. https: //doi.org/10.1145/1375581.1375584

Koushik Sen, Grigore Roşu, and Gul Agha. 2005. Detecting Errors in Multithreaded Programs by Generalized Predictive Analysis of Executions. In Formal Methods for Open Object-Based Distributed Systems, Martin Steffen and Gianluigi Zavattaro (Eds.). Springer Berlin Heidelberg, Berlin, Heidelberg, 211-226.

Yao Shi, Soyeon Park, Zuoning Yin, Shan Lu, Yuanyuan Zhou, Wenguang Chen, and Weimin Zheng. 2010. Do I Use the Wrong Definition?: DeFuse: Definition-use Invariants for Detecting Concurrency and Sequential Bugs. In Proceedings of the ACM International Conference on Object Oriented Programming Systems Languages and Applications (OOPSLA '10). ACM, New York, NY, USA, 160-174. https://doi.org/10.1145/1869459.1869474

Yannis Smaragdakis, Jacob Evans, Caitlin Sadowski, Jaeheon Yi, and Cormac Flanagan. 2012. Sound Predictive Race Detection in Polynomial Time. In Proceedings of the 39th Annual ACM SIGPLAN-SIGACT Symposium on Principles of Programming Languages (POPL '12). ACM, New York, NY, USA, 387-400. https://doi.org/10.1145/2103656.2103702

L. A. Smith, J. M. Bull, and J. Obdrzálek. 2001. A Parallel Java Grande Benchmark Suite. In Proceedings of the 2001 ACM/IEEE Conference on Supercomputing (SC '01). ACM, New York, NY, USA, 8-8. https://doi.org/10.1145/582034.582042

Francesco Sorrentino, Azadeh Farzan, and P. Madhusudan. 2010. PENELOPE: Weaving Threads to Expose Atomicity Violations. In Proceedings of the Eighteenth ACM SIGSOFT International Symposium on Foundations of Software Engineering (FSE '10). ACM, New York, NY, USA, 37-46. https://doi.org/10.1145/1882291.1882300

SPEC. 2008. SPEC releases free SPECjvm2008 benchmark. (2008). https://www.spec.org/jvm2008/press/release.html

Christoph von Praun and Thomas R. Gross. 2001. Object Race Detection. In Proceedings of the 16th ACM SIGPLAN Conference on Object-oriented Programming, Systems, Languages, and Applications (OOPSLA '01). ACM, New York, NY, USA, 70-82. https://doi.org/10.1145/504282.504288

Jan Wen Voung, Ranjit Jhala, and Sorin Lerner. 2007. RELAY: Static Race Detection on Millions of Lines of Code. In Proceedings of the the 6th foint Meeting of the European Software Engineering Conference and the ACM SIGSOFT Symposium on The Foundations of Software Engineering (ESEC-FSE '07). ACM, New York, NY, USA, 205-214. https://doi.org/10.1145/ 1287624.1287654

Chao Wang, Sudipta Kundu, Malay Ganai, and Aarti Gupta. 2009. Symbolic Predictive Analysis for Concurrent Programs. In Proceedings of the 2Nd World Congress on Formal Methods (FM '09). Springer-Verlag, Berlin, Heidelberg, 256-272. https://doi.org/10.1007/978-3-642-05089-3_17

Misun Yu, Joon-Sang Lee, and Doo-Hwan Bae. 2018. AdaptiveLock: Efficient Hybrid Data Race Detection Based on Real-World Locking Patterns. International Journal of Parallel Programming (04 Jun 2018). https://doi.org/10.1007/s10766-018-0579-5

Yuan Yu, Tom Rodeheffer, and Wei Chen. 2005. RaceTrack: Efficient Detection of Data Race Conditions via Adaptive Tracking. SIGOPS Oper. Syst. Rev. 39, 5 (Oct. 2005), 221-234. https://doi.org/10.1145/1095809.1095832

Ke Zhai, Boni Xu, W. K. Chan, and T. H. Tse. 2012. CARISMA: A Context-sensitive Approach to Race-condition Sampleinstance Selection for Multithreaded Applications. In Proceedings of the 2012 International Symposium on Software Testing and Analysis (ISSTA 2012). ACM, New York, NY, USA, 221-231. https://doi.org/10.1145/2338965.2336780 